NATURE REVIEWS DRUG DISCOVERY 13(2): 105-121 (2014) doi:10.1038/nrd4163 


\section{The Role of Ligand Efficiency Measures in Drug Discovery}

Andrew L. Hopkins, ${ }^{1}$ György M. Keserû, ${ }^{2}$ Paul D. Leeson, ${ }^{3 *}$ David C. Rees, ${ }^{4}$ Charles H. Reynolds ${ }^{5}$

1. Division of Biological Chemistry and Drug Discovery, College of Life Sciences, University of Dundee, Dundee DD1 5EH, United Kingdom. a.hopkins@dundee.ac.uk

2. Hungarian Academy of Sciences, 1025 Budapest, Pusztaszeri út 59-67, 1525 Budapest, P.O. Box 17, Hungary. keseru.gyorgy@ttk.mta.hu

3. GlaxoSmithKline, Medicines Research Centre, Gunnels Wood Road, Stevenage, Hertfordshire, SG1 2NY, United Kingdom. paul.d.leeson@GSK.com

4. Astex Pharmaceuticals , 436 Cambridge Science Park, Milton Road, Cambridge, CB4 OQA, United Kingdom. David.Rees@astx.com

5. Gfree Bio, LLC, 3805 Old Easton Road, Doylestown, Pennsylvania 18902, United States. creynolds@gfreebio.com 


\section{Summary}

- Ligand efficiency measures quantify the molecular properties, particularly size and lipophilicity, of small molecules that are required to gain binding affinity to a drug target. For example, ligand efficiency, is the binding free energy per heavy atom count ( $L E=\Delta G / H A)$ and lipophilic ligand efficiency $\left(L L E=p C_{50}\right.$ or $\mathrm{Ki}-$ cLogP/D). There are additional efficiency measures for groups in a molecule, and for combinations of size and lipophilicity.

- The application of ligand efficiency metrics has been widely reported in the selection and optimisation of fragments, hits, and leads. In particular, optimisation of lipophilic ligand efficiency shows that it is possible to increase affinity and reduce lipophilicity at the same time, even with challenging 'lipophile-preferring' targets.

- Mean ligand efficiency measures of molecules acting at a specific target, when combined with their drug-like physical properties, is a practical means of estimating target 'druggability.' This is exemplified with 480 targetassay pairs from the primary literature. Across these targets correlations between biological activity in vitro and physical properties are generally weak, showing that increasing activity by increasing physical properties is not always necessary.

- An analysis of 46 recently marketed oral drugs shows that they frequently have highly optimised ligand efficiency and lipophilic ligand efficiencies for their target. Compared with 'only-in-class' oral drugs, on average only $1.5 \%$ of all molecules per target possess superior combined ligand efficiency and lipophilic ligand efficiency values.

- Optimising ligand efficiencies based on both molecular size and lipophilicity, when set in the context of the specific target, has the potential to ameliorate the molecular inflation that pervades current practice in medicinal chemistry, and to increase the developability of drug candidates. 


\section{Biographies}

\section{Andrew L. Hopkins}

Andrew Hopkins won a British Steel scholarship to attend University of Manchester graduating with a First Class Honours in Chemistry, in 1993. He undertook a doctorate in molecular biophysics at the University of Oxford, from where he joined Pfizer, in 1998. After a career of nearly ten years in the pharmaceutical industry, Andrew moved to the University of Dundee to take up the Chair of Medicinal Informatics and SULSA Research Professor of Translational Biology, in the College of Life Sciences, University of Dundee, where he is also the Director of SULSA - the Scottish Universities Life Sciences Alliance.

\section{György M. Keserü}

György Keserü (PhD, DSc) started his career at Sanofi heading a chemistry research lab. He moved to Gedeon Richter in 1999 as the Head of Computer-aided Drug Discovery. In 2007 he was appointed as the Head of Discovery Chemistry at Gedeon Richter. He contributed to the discovery of the antipsychotic Cariprazine ${ }^{\circledR}$ that is under NDA filing. In January 2013 he became general director of the Research Center for Natural Sciences at the Hungarian Academy of Sciences. His research interests include medicinal chemistry, drug design, and in silico ADME. György has published over 160 papers and more than 10 books and book chapters.

\section{Paul D. Leeson}

Paul Leeson's 35-year industrial career began at Smith Kline and French Research Laboratories, and has taken him to Merck Sharp and Dohme, then to Wyeth (USA), and from 1997-2011, AstraZeneca, where he was head of medicinal chemistry at the Charnwood site and leader of AstraZeneca's Global Chemistry Forum. Since 2011 he has been a consultant for GlaxoSmithKline. Paul's drug discovery contributions, published in more than 150 papers, patents and book chapters, have been in the cardiovascular, neuroscience, inflammation and respiratory therapy areas. He has a special interest in compound quality in relation to pipeline attrition.

\section{David C. Rees}

David Rees joined Astex in 2003 and is Senior Vice President of Medicinal Chemistry. Prior to Astex he had 19 years' experience at Parke-Davis, Cambridge then Organon, Scotland and AstraZeneca, Sweden. In 2007 David was a co-recipient of the Royal Society of Chemistry Malcolm Campbell Memorial Prize for the discovery of Sugammadex (Bridion) which is marketed by Merck in over 40 countries. David is a co-author of over 100 publications and patents and served as President of the Organic Division of the Royal Society of Chemistry (2010-2013). 


\section{Charles H. Reynolds}

Charles Reynolds is currently President of Gfree Bio, a modeling and structure-based design company in suburban Philadelphia. Previously he was Senior Director of Discovery Technologies at Ansaris, led modeling groups at Johnson \& Johnson, and was a Research Fellow at Rohm and Haas (Dow). He received his BA from Austin College and PhD from the University of Texas at Austin. Charles has published extensively on modeling in drug discovery, and his views on the subject have been sought by organizations ranging from C\&E News to the Wall Street Journal. He is a Fellow of the American Chemical Society. 


\section{Abstract}

The judicious application of ligand or binding efficiencies, which quantify the molecular properties required to gain binding affinity for a drug target, is gaining traction in the selection and optimisation of fragments, hits, and leads. Retrospective analysis of recently marketed oral drugs shows that they frequently have highly optimised ligand efficiency values for their target. Optimising ligand efficiencies based on both molecular size and lipophilicity, when set in the context of the specific target, has the potential to ameliorate the molecular inflation that pervades current practice in medicinal chemistry, and to increase the developability of drug candidates. 


\section{Introduction}

The properties of small-molecule drugs, especially those that are orally bioavailable, are concentrated in a relatively narrow range of physicochemical space, known as 'drug-like' space. ${ }^{1,2}$ In studies of extensive datasets of small molecules, the fundamental properties of molecular size, lipophilicity, shape and polarity have been correlated, to varying degrees, with solubility ${ }^{3}$, membrane permeability ${ }^{4}$, metabolic stability, ${ }^{5,6}$ receptor promiscuity, ${ }^{7}$ in vivo toxicity, ${ }^{8,9}$ and attrition ${ }^{10,11}$ in drug development pipelines.

Lipophilicity and hydrogen bond donor count appear to be key properties, since these have remained essentially constant in oral drugs over time..$^{7,12,13,14,15}$ The median and mean molecular weight of approved drugs has risen by only around 50Da (15\%) over the past 3 decades, whilst the median and mean molecular weight of synthesized experimental compounds has risen by over 100Da (30\%). ${ }^{16}$ In contrast, the molecules being published in the literature, ${ }^{15}$ and patented by the pharmaceutical industry, ${ }^{17}$ as well as those entering clinical development pipelines, ${ }^{10}$ are more lipophilic, larger, and less three-dimensional ${ }^{14,18}$ than approved oral drugs. Yet, compounds with higher molecular weight and higher lipophilicity face a higher probability of failure at each stage of clinical development. ${ }^{10,19,20}$

The control of physical properties is dependent on the specific drug target, the mode of perturbation and the target product profile - all of which may justify developing compounds which lie beyond the norm - and on the variable drug discovery practices of originating institutions. ${ }^{7,17}$ Individual drug discovery projects often justify the pursuit of molecules that have additional risk associated with suboptimal physicochemical properties, as long as experimental project goals and target product profile criteria are met. However, viewed in aggregate at a company portfolio level, physical properties of investigational drugs can have an important influence on the overall attrition rates ${ }^{10,19,20}$ and therefore return on investment.

Three factors have been proposed to underlie the inflation in physicochemical properties ${ }^{21,22}$ of investigational drugs over the past three decades. Firstly, the discovery of initial hit compounds with inflated physical properties has been linked to the rise of high-throughput screening (HTS). ${ }^{23}$ Larger and more lipophilic compounds, potentially having greater binding affinity, are more likely to be detected in HTS assays, which are often based on a single affinity end point. Secondly, the tendency of HTS methods to identify large and lipophilic compounds is amplified by the observed tendency for the lead optimisation process to inflate physical properties. ${ }^{24,25}$ Thirdly, the portfolio of drug targets being tackled by the industry is changing towards more chemically challenging and less druggable targets. ${ }^{20}$

We believe that the obsession with potency and the co-tendency to inflate molecular properties can be remedied by monitoring and optimising ligand efficiencies instead of potency alone. Ligand efficiencies, which are measures of in vitro biological activity corrected for the physical property 'load' of the molecule, quantify how effectively the molecule uses its structural features in binding to the target. The ligand efficiency concept ${ }^{26}$ was derived from the observation of the maximum affinities achievable by ligands is -1.5 $\mathrm{kcal} / \mathrm{mol} /$ non-hydrogen atom ('heavy' stom), ignoring simple cations and anions ${ }^{27}$ and studies examining functional group binding energy. ${ }^{28}$ Kuntz et al. presented binding free energy $(\Delta \mathrm{G})$ per atom by dividing the 
free energy of binding by the number of non-hydrogen ('heavy') atoms. ${ }^{27}$ Kuntz et al. postulated the potential use of the binding energy per atom as a means to assess ligands throughout the drug discovery process. ${ }^{27}$ Transforming the data in this manner makes two assumptions. Firstly it assumes the assigns all of the intermolecular interaction to the ligand alone. Secondly, it assumes the binding energies per atom are additive. Thus for a given ligand efficiency (the gradient) there is a linear relationship between the free energy of binding and the number of heavy atoms, where it is assumed a ligand with zero atoms has zero free energy of binding at standard conditions. The most widely used ligand efficiencies are simply calculated and are summarised in Boxes 1-3; they can be applied at all stages of drug discovery, to evaluate fragments, screening hits, leads and candidate drugs. The use of molecular size, such as heavy atom count, in efficiency measures (see Box 1 ) has some caveats. ${ }^{29}$ Specifically, component atoms ( $, N, \mathrm{O}, \mathrm{S}$ and halogen) are treated equally when their sizes and binding properties are different, and some atoms in a molecule may not take part in receptor binding interactions (discussed in Box 2).

Monitoring ligand efficiency metrics during hit and lead optimisation brings into focus the price paid in physical property changes when modulating binding affinity. Depending on the binding affinity and physical properties of a lead molecule, ligand efficiencies may increase or decrease during optimisation. This is exemplified by the changes in affinity that are needed to maintain constant ligand efficiencies when substituting a lead molecule with various groups (see Box 3 and Figure 1). Applying ligand efficiency analysis has practical utility in guiding lead discovery, and more importantly lead optimisation, towards drug-like chemical space. We illustrate this with new analyses, including optimisations from the recent literature and surveys of ligand efficiencies of both drug targets and recently approved oral drugs.

\section{Binding thermodynamics and ligand efficiency}

Hit-to-lead efforts typically start from hits with micro-Molar( $\mu \mathrm{M})$ affinity or even low milli-Molar (mM) affinity in the case of fragment hits, and aim to identify sub- $\mu \mathrm{M}$ lead series with promising physicochemical and ADMET profiles that are suitable for further optimization. Improving potency increases the negative free energy of binding $(\Delta G)$, which in turn is composed of two thermodynamic quantities: binding enthalpy $(\Delta H)$ and binding entropy $(\mathrm{T} \Delta \mathrm{S})$.

Predicting how structural modifications will affect enthalpy or entropy of binding is extremely difficult, particularly given the well-known phenomenon of entropy-enthalpy compensation, but there are some general trends. ${ }^{30,31}$ Optimisation of specific polar interactions between the target binding site and ligand is usually associated with a more promising physicochemical profile (lower lipophilicity) and improvements in binding enthalpy. In contrast, increasing ligand size and lipophilicity to generate non-specific interactions often leads to entropy-driven improvements in affinity, ${ }^{32}$ while specific lipophilic interactions can increase or decrease binding enthalpy. The entropy-driven approach to improving affinity is probably a factor in the well-documented inflation of physicochemical properties in optimisation. $22,23,24,25$ 
Although this picture is qualitative, thermodynamics-based optimizations have been the subject of increasing recent interest. ${ }^{33,34}$ Isothermal titration calorimetry (ITC) allows the determination of $\Delta \mathrm{H}$ and $\mathrm{T} \Delta \mathrm{S},{ }^{35}$ and ITC data from more than 700 protein-ligand complexes, obtained from a literature survey covering both medicinal chemistry and natural-product derived ligands, has been analysed. ${ }^{36}$ In contrast to binding free energy, ${ }^{27}$ the binding enthalpy decreases with increasing molecular size at heavy atom count of 25 or greater. ${ }^{37}$

LE decreases with increasing number of heavy atoms (see Box 2 and supplementary Figure S2). ${ }^{38,39}$ It has been suggested that a contributing factor to the fall-off typically observed for LE in larger ligands might be due to less favorable binding entropies for larger, more flexible ligands. ${ }^{40}$ However, in an analysis of the number of energetically accessible conformations for several thousand ligands of varying sizes and affinities no trend could be found for increased conformational entropies as ligand size increased. ${ }^{39}$ It is clear from this analysis that many large molecules are much more conformationally constrained than might be expected from overall size or a simple count of rotatable bonds. It also seems likely that any effect of conformational entropy upon ligand binding may well be swamped by other contributions to overall $\mathrm{T} \Delta \mathrm{S}$ of binding. ${ }^{40}$

Thermodynamic data allows individual ligand enthalpic and entropic efficiencies per heavy atom to be calculated. ${ }^{35}$ A size independent version of enthalpic efficiency $(\mathrm{SIHE})^{36}$ can also be used. Analysis of experimental ITC data ${ }^{33,41}$ across a wide range of chemotypes shows that the average entropy efficiency is little changed with size (supplementary Figure S1), but narrows in moving from small to large ligands. ${ }^{42}$ By contrast, the enthalpy efficiencies show a much more dramatic trend with increasing size, which is similar to the trend seen with the overall $L E$ values (ie derived from $\Delta G$ ). Very favorable enthalpy efficiencies are common for small ligands, but the average, and most favorable, enthalpy efficiencies for larger ligands are systematically reduced (see supplementary Figure S1). Hence the overall trend in $\Delta \mathrm{G}$ related LE is mainly a consequence of enthalpy $(\Delta \mathrm{H})$ efficiency. ${ }^{40,42}$ These results suggest that the effects of conformational entropy may not be as significant as is commonly believed. In contrast to size, both lipophilic enthalpy and lipophilic entropy efficiencies tend to decrease with increasing lipophilicity (see Supplementary Figure S1). For individual protein targets, lipophilic ligand efficiency (LLE or LipE, Box 1) might be a more useful surrogate than LE or LELP for the enthalpic component of ligand binding, especially under circumstances where structurally similar series, such as matched molecular pairs, display similar specific lipophilic binding interactions. $^{43}$

Optimisation of enthalpy-driven binding efficiency appears to be an attractive strategy, ${ }^{37}$ but it is not yet clear that this will lead to less attrition in drug candidate pipelines. Analysis of four targets from the literature suggests that as well as enthalpy efficiency, lipophilic ligand efficiency measures can provide practical guidance in designing ligands with improved molecular properties, ${ }^{44}$ as discussed in the next section. 


\section{Lipophilic ligand efficiency}

The mean lipophilicity of marketed drugs - measured by the partition coefficient ( $\log \mathrm{P})$ or the distribution coefficient ( $\log D)$ - has changed little over several decades. ${ }^{7,12,13,14,15}$ This important observation implies that lipophilicity is a fundamental property impacting the progress of drug discovery programs and the developability of identified candidates. In contrast, it has been observed that the physicochemical properties of analogues in a chemical series, including molecular weight and lipophilicity, often increase during optimisation from hit to lead ${ }^{23}$ and lead to candidate. ${ }^{24,25}$

The concept of employing 'minimal hydrophobicity' in drug design is not new, ${ }^{45}$ and is supported by a wealth of recent evidence ${ }^{46}$ showing that lipophilicity has an important influence on drug-like properties. In addition to its connection to solubility, ${ }^{3}$ lipophilicity impacts most of the ADME parameters and also toxicity properties such as hERG liability, phospholipidosis, CYP inhibition and receptor promiscuity. ${ }^{46}$ Due to its central role in pharmacokinetics and safety most of the empirical ADMET guidelines include lipophilicity, such as Lipinki's rule of five on absorption, ${ }^{47}$ the GSK $4 / 400$ guideline on ADMET properties, ${ }^{6}$ and the Pfizer $3 / 75$ guideline on toxicological outcomes. ${ }^{8}$

In addition to influencing ADMET parameters, lipophilicity (LogP or LogD) is also one of the key factors determining binding affinity to drug target proteins. High target potency combined with high lipophilicity may therefore also increase the risk of ADMET related attrition. ${ }^{2}$ As a result, medicinal chemistry optimization needs to be balanced and multidimensional, ${ }^{37}$ a difficult task that can be assisted by the use of efficiency metrics to control lipophilicity. Lipophilic ligand efficiency ( $\operatorname{LLE}^{7}$ or LipE, ${ }^{43}$ Box 1 ) is a simple but important index combining in vitro potency and lipophilicity. A molecule with LLE $=0$ based on cLogP, where target affinity = cLogP, can be thought of as having the same 'affinity' for its target as it does for 1-octanol, whereas a drug candidate with LLE $=6$ has a one million-fold preference for its target versus 1-octanol. Negative LLE values are clearly unfavourable. Based on the properties of an average oral drug, with cLogP $\sim 2.5-3.0$ and potency in the range $\sim 1-10 \mathrm{nM}$, an ideal LLE value for an optimised candidate drug is $\sim 5-7$ or greater. ${ }^{7}$ Fragments or lead-like molecules used as chemical starting points generally cannot possess druglike LLE values because they are not potent enough. Hits with LLE $\leq 2$ are commonly found from high throughput screening (i.e. $\geq 1 \mathrm{UM}$ affinity with $\operatorname{cLogP} \sim 4^{23}$ ), and these will have to be improved by $\geq \sim 3$ LLE units during optimisation to a candidate. Additional metrics such as LE are useful to deal with molecular size in fragment-to-hit and hit-to-lead optimisations.

Combining both size and lipophilicity into a single efficiency index is useful at the hit identification and hit-tolead stages, and in fragment based drug discovery (FBDD, see the next section). Two such parameters lipophilicity corrected ligand efficiency (LELP) ${ }^{23}$ and $\operatorname{LLE}_{A T}{ }^{48}-$ have been developed (Box 1). While the full range of optimal LELP values ${ }^{23}$ is -10 to +10 , a desirable hit or lead against a tractable target in the early optimization stage, having LE > 0.40-0.45 and CLogP 0-3, will have LELP 0-7.5. LLE $E_{A T}$ was derived from experience in FBDD, and is scaled to be comparable in magnitude to $L E{ }^{48}$ Both LELP and $L_{L E}$ AT can also be applied in lead optimisation. 
It has been shown ${ }^{44}$ that increasing lipophilic efficiency, using LLE and LELP values, is associated with improved ADMET and safety endpoints, probably as a result of both lowered lipophilicity and increased specificity. In one study, development candidates were successfully discriminated from marketed drugs by LELP but not by LE and LLE alone. ${ }^{49}$ In addition, compilations of hits, leads, successful leads (those that produced marketed drugs ${ }^{50}$ ) and drugs are differentiated by their mean LLE and LELP values ${ }^{44}$ (potency, LE, LLE and LELP values for these compound sets are given in supplementary Table S1). An analysis presented below in the section 'ligand efficiencies of oral drugs' shows that using LE and LLE together can differentiate marketed drugs from other molecules acting at the same target.

The recent medicinal chemistry literature contains an increasing number of examples where LLE (or LipE) has been explicitly employed in the optimisation process; 59 examples, covering 47 different molecular targets, are summarized in Figure 2 (see supplementary spreadsheet for details). For each example, in vitro activities, LLE and LE values of the starting points and optimised molecules were collected. The median changes in pActivity and LLE were +1.22 and +1.96 respectively ( $p<0.001$ from matched pair analysis). Median values of $\operatorname{LELP}(-2.74)$ and $\operatorname{LLE}_{\mathrm{AT}}(+0.066)$ were similarly significantly reduced and increased, respectively. These results show that focussing on LLE in lead optimisation facilitates discovery of molecules with increased binding affinity, without necessarily increasing lipophilicity at the same time. In fact, 48 of the 59 examples increased LLE in the optimisation process, even though for many of the examples cited, optimising properties in addition to binding was undertaken.

The importance of lipophilicity control in optimisation is further exemplified in an analysis ${ }^{50}$ of 60 recently marketed drugs and their leads, which showed that mean lipophilicity stayed constant during the optimisation process, while mean potency, and therefore mean LLE, increased by $\sim 2$ log units. For these 60 drug examples, ${ }^{50}$ high drug LLE is linked to lower lead CLogP, the straight line fit being LLE drug $=7.93-0.78 \mathrm{x}$ CLogP lead $\left(n=60, r^{2}=0.47, p<0.0001\right)$. In the recent 59 LLE-aware optimisations in Figure 2 , the same trend is present but as expected is much less pronounced: optimised LLE $=6.15-0.30 \times \operatorname{lead} \operatorname{cLogP}\left(n=59, r^{2}=\right.$ $0.077, p=0.033$ ). Achieving high LLE values in optimised molecules is therefore more likely when starting with low cLogP leads (ideally $<3$ ). These observations on newer drugs and on current practices contrast with earlier literature studies, ${ }^{24,25,51}$ largely pre-dating the application of ligand efficiency concepts, in which lipophilicity, on average, increased during optimisation. We conclude that optimisation of lipophilic ligand efficiencies will guide projects towards potent molecules with lowered lipophilicity, which will in turn improve the developability of emerging candidate compounds.

It is interesting to note that mean LE did not change in either the lead to drug optimisations ${ }^{50}$ or those in Figure 2. The conservation of LE during lead optimisation supports the original premise of LE as a metric to aid the selection of leads, as well as to compare compounds for further optimisation at the hit selection stage. $^{20,26}$ In contrast, median SILE (size-independent ligand efficiency, Box 2) values were significantly increased by $+0.48(p<0.001)$ in the Figure 2 optimisations. SILE is therefore a metric that could be useful for differentiating molecules in the physical property ranges where lead optimisation commonly takes place. 


\section{FBDD and ligand efficiency}

An important emerging area for the application of ligand efficiency metrics is in fragment based drug discovery (FBDD). In the last decade, FBDD has been used to discover several compounds that have progressed into clinical trials ${ }^{52}$ and onto the market. ${ }^{53}$ Fragments are compounds with molecular weights between 100 and $250 \mathrm{Da}$ and are small relative to conventional compounds screened in HTS. As a consequence of their small size, fragment hits commonly have low binding affinities, usually in the range 1 $\mathrm{mM}-10 \mu \mathrm{M}$. Ligand efficiency (LE) is a useful metric to normalise the affinities of hits to identify the best starting points for optimisation, i.e. those with the highest LE values, all else being equal.

Despite their low affinities, fragment hits selected for further optimisation often have generally good (>0.4) to excellent LE values. Growing these fragments into leads is a challenge often requiring the addition of $>\sim 15-20$ heavy atoms ( 200-250 Da increase in molecular weight) to increase affinity by several orders of magnitude ( $\mathrm{mM}$ or $\mu \mathrm{M}$ to $\mathrm{nM}$ ). This presents an opportunity to use the various ligand (Box 1 ) and group efficiency (GE, Box 3$)^{54}$ metrics to carefully control chemical properties. This approach to FBDD, when practised by specialist teams, can result in optimised molecules with improved drug-like properties. ${ }^{17}$

An example FBDD programme targeted at the chaperone protein, HSP90, a promising anticancer target ${ }^{55}$, illustrates the general principle of only adding atoms to the starting fragment providing the desired increase in binding affinity (Figure 3a). This example is a showcase, as six heavy atoms are added and the affinity increases by 6 orders of magnitude $(0.79 \mathrm{mM}$ to $0.70 \mathrm{nM}) .{ }^{56,57}$ Careful attention to the experimentally determined X-ray crystal structure and overall chemical properties were critical during this fragment-to-lead optimisation. The metric $L L E_{A T}$ is useful during this stage because it represents LLE normalised for size (see Box 1) and helps to ensure that affinity increase is not unduly driven by non-specific lipophilic interactions. LE metrics are, by definition, useful for in vitro binding affinity but the advantage of starting in vivo optimisation with high LE is that additional atoms can be added to the lead to optimise in vivo efficacy, pharmacokinetics and safety and still provide a clinical candidate that is 'drug-like' in terms of molecular weight and lipophilicity.

Group Efficiency (GE, Box 3) is also particularly useful when growing fragments because it focuses on the efficiency of atoms added to the original molecule. In the HSP90 example, the GEs for each group in the final molecule are determined by comparing matched pairs of related compounds. The groups with high GE are those that appear in the X-ray crystal structure to form clear binding interactions. The GE values for the different parts of the HSP90 inhibitor, AT13387, were determined retrospectively by comparing the binding affinities of closely related compounds (Figure 3b; see Box 3 for the method used). Several other published examples of fragment optimisations and the use of LE metrics have been reviewed. ${ }^{58,59}$ 


\section{Ligand efficiencies of oral drugs}

Target classes (for example GPCRs, kinases, proteases, nuclear hormone receptors) display differing molecular properties amongst their small molecule ligands. ${ }^{17,20,51,60}$ Ligand efficiency measures (LE and LLE) can be used in conjunction with physical properties (cLogP and HA) to assess the relative 'druggability' of human drug targets and target classes, by analysis of their known ligands (see Box 4 and Figure 4). The variability in ligand efficiencies and physical properties of targets evident in Figure 4 is a consequence of the inherent differences in the relative 'druggability' of targets combined with varying practices in drug discovery ${ }^{7,17}$ leading to published active compounds.

Drug molecules are the final result of the exhaustive optimisation of chemical, biological, toxicological, pharmaceutical and clinical profiles of lead compounds. Can ligand efficiency metrics help distinguish drugs from non-drugs? We compared LE and LLE values of recently marketed drugs in comparison with other molecules with reported activity at the same target (see Figure 5). The dataset was compiled from searching CHEMBL for compounds with published human target affinity in vitro ( $\mathrm{IC}_{50}, \mathrm{Ki}$ or $\mathrm{EC}_{50}$ values), and calculating LE and LLE values (Box 1). The percentage of all compounds at each target where both LE and LLE values are superior to the oral drug was determined. This approach weights LE and LLE equally and to achieve a low percentage score (the y axis of Figure 5) a drug must rank highly on one or both of these efficiency measures. A group of first-in-class, and currently 'only-in-class' drugs acting on single targets, stands out as having notably better combined LE and LLE values versus other compounds acting on the same primary targets. These drugs (Figure 5) include aprepitant (NK1 antagonist), aliskiren (renin inhibitor), vorinostat (HDAC1 inhibitor), maraviroc (CCR5 antagonist, see also Figure 6a), lorcaserin (5-HT2c agonist), roflumilast (PDE4A inhibitor), ruxolitinib (JAK2 kinase inhibitor), tofacitinib (JAK3 kinase inhibitor), vismodegib (SMO/hedgehog inhibitor), fingolimod (S1P1 inhibitor; the active form is the S-phosphorylated metabolite) and ticagrelor (P2Y12 antagonist). While these targets occupy varying absolute LE/LLE/physical property space, decreasing in relative druggability from 5-HT2c and HDAC1 to renin and CCR5, the median percentage molecules of per target with superior combined LE and LLE values for these 'only-in-class' drugs is just $1.5 \%$.

Amongst other targets in Figure 5, two NS3 protease inhibitors, boceprevir and telaprevir, were both approved by the FDA in May 2011 for treatment of hepatitis C virus. NS3 protease is a challenging target for good inhibitor physicochemical properties, but boceprevir is clearly highly optimised in the class, with only $1.0 \%$ of NS3 inhibitors having better combined LE and LLE values. For telaprevir, which is elaborated with a bicyclic structural moiety that is absent in boceprevir, 38\% of NS3 inhibitors have a better LE and LLE profile. Both drugs are covalent inhibitors expressing slow dissociation kinetics and requiring high doses (telaprevir $750 \mathrm{mg}$ and boceprevir $800 \mathrm{mg}$, both three times daily). However, the physical properties of telaprevir result in very low solubility, ${ }^{61}$ requiring a non-standard formulation, and it also carries a boxed warning on the label for risk of serious skin reactions. 
Many anti-cancer drugs inhibiting kinases show non-optimal LE and LLE values for their targets (Figure 5). The median percentages of compounds per target that have better LE and LLE values in Figure 5 is $22 \%$ for kinase inhibitors versus $2.7 \%$ for the other target classes. An example is VEGF2 kinase, where three drugs are non-optimal (sunitinib, sorafenib and pazopanib), but the newest drug (axitinib) is highly optimised. A recent study of clinical VEGFR inhibitors emphasises the value of lipophilic efficiency, showing that LLE correlates with kinase selectivity as well as clinical efficacy, ${ }^{62}$ the latter probably driven by relative exposure levels. In the case of EGFR kinase, all four drugs have non-optimal LE and LLE values. The difference between kinase inhibitor drugs and other classes may be due to the primary pursuit in the discovery phase of selectivity ${ }^{63}$ versus other kinases, and the acceptance of higher safety risk: benefit profiles for treatment of cancer. Just one kinase inhibitor drug, the JAK3 inhibitor tofacitinib, ${ }^{64}$ is aimed at a non-cancer indication, and it has the best combined LE and LLE values of all reported JAK3 ligands. Amongst drug families (Figure 5), highly optimal LE and LLE values are seen for all three Factor Xa inhibitors ${ }^{65}$ rivaroxaban, apixaban and edoxaban (respectively $0.2 \%, 0.9 \%$ and $1.1 \%$ of reported molecules with better LE and LLE), and the non-prodrug parent molecules of the thrombin inhibitors melagatran and dabigatran ( $1.1 \%$ and $6.5 \%$ better respectively). For DPP-IV inhibitors, improvements in the most recently approved molecule (saxagliptin) are seen versus the first in class molecule (sitagliptin); a similar trend is seen for endothelin A antagonists.

\section{Application of efficiency metrics in optimisation}

The oral drug analysis in Figure 5 indicates that plotting LE versus LLE will be useful for analysis and tracking progress of hit to lead and lead optimisation projects, as well as for evaluation of the relative properties of clinical candidates. In LE versus LLE plots, there is some level of redundancy in that the potency term appears on both axes. The correlation between LE and LLE will increase when both size and lipophilicity influence potency similarly. However, potency versus property relationships often have low correlation coefficients and vary according to target and ligand chemotype (see Figure 4c and d). The purpose of using LE and LLE together is to illustrate the value to the drug designer of this visualisation, rather than to establish quantitative correlations. In addition to LE versus LLE, separately examining the independent component parameters by constructing plots of potency versus both HA (or molecular weight) and lipophilicity (cLogP or LogD) is essential. An example of LE versus LLE is shown in Figure 6a for the chemokine CCR5 receptor, ${ }^{66}$ where there has been an intensive effort to find antagonists for treatment of HIV and rheumatoid arthritis. CCR5 also proved to be a useful target to assess different drug design practices in the pharmaceutical industry. ${ }^{7}$ The only molecule to date to be approved, for HIV, is maraviroc, which has improved combined LE and LLE versus the other CCR5 antagonist HIV clinical candidates (Figure 6a). A major challenge with CCR5 ligands has been reducing unwanted cardiovascular risk because of inhibition of the hERG ion channel. This can be accomplished by reducing lipophilicity in combination with structure-activity optimisation. ${ }^{67}$

One of the most challenging targets of all in terms of obtaining drug-like physical properties is the cholesteryl ester transfer protein (CETP), ${ }^{68}$ an atherosclerosis target where inhibition raises HDL-c levels. ${ }^{69}$ CETP has 
highly lipophilic cholesteryl ester and triglyceride as co-substrates, and inhibitors of this target, including four molecules that have reached late stage clinical trials, have low LE and very low LLE values (Figure 6b shows LE versus LLE and $\mathrm{pIC}_{50}$ versus cLogP for CETP inhibitors). Nevertheless, high throughput screening has identified a class of benzoxazoles, ${ }^{70,71,72,73}$ apparently not yet fully optimised, with markedly improved LE and LLE values versus the clinical candidates. In addition, further optimisation of the highly lipophilic candidate torcetrapib (which was discontinued in Phase III) has resulted in analogues with significantly lowered lipophilicity yet with equal potency, ${ }^{74}$ increasing LLE by $\sim 4$ units with no adverse effect on LE (compare compounds $\mathbf{7}$ and 13, Figure 6b). In this example, a stated strategy was to 'mitigate lipophilicity" ${ }^{74}$ which was successfully achieved, although no LogP/D or LLE values were actually cited. Explicit use of quantitative LE and LLE measures during optimisation, assisted by visualisations of LE versus LLE and potency versus lipophilicity and size, is the approach we recommend. By contrast, optimisation of a class of diphenylpyridylethanamine CETP inhibitors, ${ }^{75}$ conducted without obvious consideration of ligand efficiency or lipophilicity, resulted in a lead compound with an unfavourable negative LLE value (compound 20 in ref. 75: $\mathrm{pIC}_{50}$ 7.44, cLogP 8.96, LLE = -1.52).

The cannabinoid CB1 receptor is another target with highly lipophilic endogenous ligands, where it has been challenging to control physical properties. Specific application of the LLE concept has been successfully used in optimisation of both CB1 antagonists ${ }^{76}$ and agonists ${ }^{77}$ (Figure 7). In these examples, unpromising LLE values of close to zero in the lead compounds were impressively increased by $\sim 5$ units, while retaining constant LE values.

We can conclude that, even on the least tractable targets that appear to have highly lipophilic small molecule binding sites, seeking compounds with improved properties is facilitated when the focus is placed on improving ligand efficiencies. The direction of optimisation in LE versus LLE plots is to the 'north east' and in potency versus lipophilicity (cLogP or LogD) or size plots, optimisation should proceed to the 'north west.' In either case, property-based design should seek to extend the boundaries of these plots to new, unoccupied territory. In addition to LE versus LLE and potency versus size and lipophilicity, other visualisations useful for optimisation purposes include: potency versus LE, LLE, LELP, and LLE $_{A T} ;$ $L E$ versus CLogP or LogD; LLE versus $\mathrm{HA}$ or molecular weight; and the property forecast index ${ }^{3}$ (PFI= LogP or LogD + aromatic ring count) versus $\mathrm{LLE}_{\mathrm{AT}}{ }^{65}$ Lipophilic efficiency parameters for agonists can be adjusted if required to take intrinsic activity into account. $^{78}$

Overall, the retrospective analysis of successful drugs indicates that optimising LE and LLE in concert is an important success factor for hit and lead optimisation in drug discovery projects. Controlling lipophilicity is at the heart of successful optimisation. Most of the medicinal chemistry designs employed in the examples shown in Figure 2 leading to LLE increases did not require radical structural changes such as new core scaffolds. The examples of compound $\mathbf{7}$ to compound $\mathbf{1 3}$ in Figure 6b, and those in Figure $\mathbf{7}$ are typical. Tactics (see Figure 7) include i) replacing carbon atoms with oxygen or nitrogen atoms (especially converting 
phenyl rings to heteroaromatic rings), ii) adding polar substitution, iii) removing or replacing lipophilic substituents and templates and iv) controlling growth of heavy atom count. Incorporating polar functional groups which both lower lipophilicity and increase or retain binding affinity is probably the result of new polar ligand-receptor contacts ${ }^{79}$ (for example hydrogen bonds), which will radically alter structure-affinity relationships, and consequently provide new optimisation opportunities. That said, finding the appropriate regions of a lead molecule which can be changed in these ways may be the biggest challenge - highly lipophilic hits or leads resistant to this approach should be quickly dropped. Finally, LLE correlates positively with the drug efficiency index (DEI).$^{80} \mathrm{DEl}$ is a very useful metric for application in lead optimisation projects, since it combines the estimated fraction of the dose that is available free in plasma with in vitro potency.

\section{Conclusion}

Ligand efficiency measures provide estimates of the physical properties used to gain affinity for a drug target. The judicious use of efficiency metrics in optimisation instead of potency alone is recommended at all stages of drug discovery, starting with the selection of a fragment or a screening hit. Oral drugs, especially those that are first in class in therapy areas with stringent risk/benefit requirements, frequently have highly optimised ligand efficiency values for their target. A key consideration for drug designers is awareness of the changes in potency required to keep ligand efficiencies at least constant when altering a lead structure. When all published molecules with activity at specific targets are considered, high correlations between affinity and size or lipophilicity are not frequent, therefore it is not always necessary to increase these physical properties to increase affinity. The tendency to increase lipophilicity during optimisation of individual series of molecules can be countered by focusing on lipophilic efficiency. This is becoming recognised as a key strategy in lead optimisation ${ }^{43,81}$ and has been successfully employed on many different targets. Application of ligand efficiency principles, combined with synthesis of compounds with acceptable drug-like physical properties, are indicated as key elements of best practice in drug design. We believe this approach is applicable to any target containing a bone fide small molecule binding site. Finally, we note that the hypothesis proposed by Hansch and co-workers a quarter of a century ago embraced the concept of lipophilic ligand efficiency, and is withstanding the test of time: "without convincing evidence to the contrary, drugs should be made as hydrophilic as possible without loss of efficacy." 45

\section{Acknowledgment}

We thank Nicola Richmond (GSK) for discussions on derivation of efficiency metrics, Glyn Williams (Astex) for fruitful discussion on binding thermodynamics, Rob Young (GSK) for discussions on aromaticity and drug efficiency metrics, and AstraZeneca for provision of the GVK BIO database. 



\section{References}

1. Leeson, P. D.; Oprea, T. I. Drug-like physicochemical properties. RSC Drug Discovery Series 13 (Drug Design Strategies), 35-59 (2012).

2. Gleeson, M. P., Hersey, A., Montanari, D. \& Overington, J. Probing the links between in vitro potency, ADMET and physicochemical parameters. Nature Rev. Drug Discov. 10, 197-208 (2011).

3. Young, R. J., Green, D. V., Luscombe, C. N., Hill, A. P. Getting physical in drug discovery II: the impact of chromatographic hydrophobicity measurements and aromaticity. Drug Discov. Today 16, 822-830 (2011).

4. Waring, M. Defining optimum lipophilicity and molecular weight ranges for drug candidates-Molecular weight dependent lower logD limits based on permeability. Bioorg. Med. Chem. Lett. 19, 2844-2851 (2009).

5. Johnson, T.W. et al. Using the Golden Triangle to optimize clearance and oral absorption. Bioorg. Med. Chem. Lett. 19, 5560-5564 (2009).

6. Gleeson, M. P. Generation of a set of simple, interpretable ADMET rules of thumb. J. Med. Chem. 51, 817834 (2008).

7. Leeson, P.D., Springthorpe B. The influence of drug-like concepts on decision-making in medicinal chemistry. Nature Rev. Drug Discov. 6, 881-890 (2007).

\section{Lipophilic ligand efficiency (LLE or LipE) proposed as a measure of specificity.}

8. Hughes, J. D., Blagg, J., Price, D. A., Bailey, S., Decrescenzo, G. A., Devraj, R. V., Ellsworth, E., Fobian, Y. M., Gibbs, M. E., Gilles, R. W., Greene, N., Huang, E., Krieger-Burke, T., Loesel, J., Wager, T., Whiteley, L., Zhang, Y. Physiochemical drug properties associated with in vivo toxicological outcomes. Bioorg. Med. Chem. Lett. 18, 4872-5 (2008).

9. Luker, T., Alcaraz, L. Chohan, K. K., Blomberg, N. Brown, D. S., Butlin, R. J., Elebring, T., Griffin, A. M., Guile, S., St-Gallay, S., et al. Strategies to improve in vivo toxicology outcomes for basic candidate drug molecules Bioorg. Med. Chem. Lett. 21, 5673-5679 (2011).

10. Wenlock, M. C., Austin, R.P., Barton, P., Davis, A.M., Leeson, P.D. A comparison of physiochemical property profiles of development and marketed oral drugs J. Med. Chem. 46, 1250-6 (2003).

11. Leeson, P. D., Empfield, J. R. Reducing the risk of drug attrition associated with physicochemical properties. Ann. Rep. Med. Chem. 45, 393-407 (2010).

12. Leeson, P. D., Davis, A. M. Time-related differences in the physical property profiles of oral drugs. J. Med. Chem. 47, 6338-48 (2004).

13. Proudfoot, J. R. The evolution of synthetic oral drug properties. Bioorg. Med. Chem. Lett. 15, 1087-1090 (2005).

14. Leeson, P. D., St-Gallay, S. A., Wenlock, M. C. Impact of ion class and time on oral drug molecular properties MedChem Comm. 2, 91-105 (2011).

15. Walters, W. P., Green, J., Weiss, J. R., Murcko, M. A. What do medicinal chemists actually make? A 50-year retrospective. J. Med. Chem. 54, 6405-16 (2011).

16. Bickerton, G.R., Paolini, G.V., Besnard, J., Muresan, S., Hopkins, A.L. Quantifying the chemical beauty of drugs. Nature Chem. 4, 90-8 (2012).

17. Leeson, P. D., St-Gallay, S. A. The influence of the 'organizational factor' on compound quality in drug discovery. Nature Rev. Drug Discov. 10, 749-65 (2011).

18. Lovering, F., Bikker, J., and Humblet, C. Escape from flatland: increasing saturation as an approach to improving clinical success. J. Med. Chem. 52, 6752-6756 (2009).

19. Blake, J.F. Examination of the computed molecular properties of compounds selected for clinical development. Biotechniques Suppl, 16-20 (2003).

20. Paolini, G.V., Shapland, R.H., van Hoorn, W.P., Mason, J.S., Hopkins, A.L. Global mapping of pharmacological space. Nature Biotechnol. 24, 805-15 (2006).

21. Keserú, G. M. 5th Drug Design Lead Discovery Conference 2009: lead finding strategies and optimization case studies. Drugs Future 35, 143-153 (2010).

22. Hann, M. M. Molecular obesity, potency and other addictions in drug discovery. MedChemComm 2, 349-355 (2011).

23. Keserü, G. M, Makara, G. M. The influence of lead discovery strategies on the properties of drug candidates. Nature Rev. Drug Discov. 8, 203-12 (2009).

24. Oprea, T.I., Davis, A.M., Teague, S.J., Leeson, P.D. Is there a difference between leads and drugs? A historical perspective. J Chem Inf Comput Sci. 41, 1308-15 (2001). 
25. Hann, M.M., Leach, A.R., Harper, G. Molecular complexity and its impact on the probability of finding leads for drug discovery. J Chem Inf Comput Sci. 41, 856-64 (2001).

Explains why small compounds (eg fragments) have higher probability than larger compounds (eg HTS library) of binding to protein targets.

26. Hopkins, A. L., Groom, C.R., Alex, A. Ligand efficiency: a useful metric for lead selection. Drug Discov. Today 9, 430-1 (2004).

Ligand efficiency (LE) proposed as a measure to help prioritise screening hits.

27. Kuntz, I. D.; Chen, K.; Sharp, K. A.; Kollman, P. A. The maximal affinity of ligands. Proc. Natl. Acad. Sci. U. S. A. 96, 9997-10002 (1999).

Seminal article defining LE values ( $\mathrm{kcal} / \mathrm{mole} / \mathrm{heavy}$ atom) and laying the foundations for derivation of efficiency metrics.

28. Andrews, P. R.; Craik, D. J.; Martin, J. L. Functional group contributions to drug-receptor interactions. J. Med. Chem. 27, 1648-57 (1984).

29. Shultz, M.D., Setting expectations in molecular optimizations: strengths and limitations of commonly used composite parameters, Bioorg. Med. Chem. Lett. 23, 5980-5991 (2013).

30. Freire, E. Do enthalpy and entropy distinguish first in class from best in class? Drug Discov. Today 13, 869-74 (2008).

31. Ferenczy, G. G., Keserü, G. M. Thermodynamics of ligand binding. in Physico-Chemical and Computational Approaches to Drug Discovery (Eds. Luque, J., Barril, X.), Royal Society of Chemistry, London, 2012.

32. Olsson, T, S. G., Williams, M. A., Pitt, W. R.; Ladbury, J. E. The Thermodynamics of Protein-Ligand Interaction and Solvation: Insights for Ligand Design. J. Mol Biol. 384, 1002-1017 (2008),

33. Freire, E. A thermodynamic approach to the affinity optimization of drug candidates. Chem. Biol. Drug Des. 74, 468-72 (2009).

34. Ferenczy, G. G., Keserü, G. M. Thermodynamics guided lead discovery and optimization. Drug Discov. Today 15, 919-932 (2010).

35. Ladbury, J. E.; Klebe, G.; Freire, E. Adding calorimetric data to decision making in lead discovery: a hot tip. Nature Rev. Drug Discov. 9, 23-7 (2010).

36. Ferenczy, G. G., Keserü, G. M. Enthalpic efficiency of ligand binding. J. Chem. Inf. Mod. 50, 1536-1541 (2010).

37. Hann, M. M.; Keserü, G. M. Finding the sweet spot - the role of nature and nurture in medicinal chemistry. Nature Rev. Drug Discov. 11, 355-365 (2012).

38. Reynolds, C. H.; Bembenek, S. D.; Tounge, B. A. The role of molecular size in ligand efficiency. Bioorg. Med. Chem. Lett.17, 4258-4261 (2007).

39. Reynolds, C. H., Tounge, B.A., Bembenek, S.D. Ligand binding efficiency: trends, physical basis, and implications. J. Med. Chem. 51, 2432-8 (2008).

Ligand efficiency (LE) has a significant size-dependence that can be explained in terms of simple molecular principles.

40. Loving, K.; Alberts, I.; Sherman, W. Computational approaches for fragment-based and de novo design. Curr. Top. Med. Chem. 10, 14-32 (2012).

41. Liu, T.; Lin, Y.; Wen, X.; Jorissen, R. N.; Gilson, M. K. BindingDB: a web-accessible database of experimentally determined protein-ligand binding affinities. Nucl. Ac. Res. 35, D198-D201 (2007).

42. Reynolds, C. H., Holloway, M. K. Thermodynamics of Ligand Binding and Efficiency. ACS Med. Chem. Lett. 2, 433-7 (2011).

43. Shultz, M.D., The thermodynamic basis for the use of lipophilic efficiency (LipE) in enthalpic optimizations, Bioorg. Med. Chem. Lett. 23, 5992-6000 (2013).

44. Tarcsay, A., Nyiri, K., Keserü, G. M. Impact of Lipophilic Efficiency on Compound Quality. J. Med. Chem. 55, 1252-1260 (2012). 
45. Hansch, C., Bjoerkroth, J. P., Leo, A. Hydrophobicity and central nervous system agents: on the principle of minimal hydrophobicity in drug design. J. Pharm. Sci. 76, 663-87 (1987).

46. Waring, M. Lipophilicity in drug discovery Exp. Opin. Drug Disc. 5, 235-248 (2010).

47. Lipinski, C. A., Lombardo, F., Dominy, B. W., Feeney, P. J. Experimental and Computational Approaches to Estimate Solubility and Permeability in Drug Discovery and Development Settings. Adv. Drug Deliv. Rev. 23, 325 (1997).

48. Mortenson, P. N., Murray, C.W. Assessing the lipophilicity of fragments and early hits. J Comput Aided Mol Des. 663-7 (2011).

49. Wager, T. T., Chandrasekaran, R. Y., Hou, X., Troutman, M. D., Verhoest, P. R., Villalobos, A., Will, Y. Defining Desirable Central Nervous System Drug Space through the Alignment of Molecular Properties, in Vitro ADME, and Safety Attributes. ACS Chem. Neurosci. 1, 420-434 (2010).

50. Perola, E. An Analysis of the Binding Efficiencies of Drugs and their Leads in Successful Drug Discovery Programs. J. Med. Chem. 53, 2986-2997 (2010).

\section{Analysis of leads of 60 recently launched drugs showed mean potency and lipophilic ligand efficiency (LLE or LipE) increased in ultimately successful optimisations, whereas mean lipophilicity did not.}

51. Morphy, R. The Influence of Target Family and Functional Activity on the Physicochemical Properties of PreClinical Compounds. J. Med. Chem. 49, 2969-2978 (2006).

52. Baker, M. Fragment-based lead discovery grows up. Nature Rev. Drug Discov. 12, 5-7 (2013).

53. Tsai, J., Lee, J.T., Wang, W., Zhang, J., Cho, H., Mamo, S., Bremer, R., Gillette, S., Kong, J., Haass, N.K., Sproesser, K., Li, L., Smalley, K.S., Fong, D., Zhu, Y.L., Marimuthu, A., Nguyen, H., Lam, B., Liu, J., Cheung, I., Rice, J., Suzuki, Y., Luu, C., Settachatgul, C., Shellooe, R., Cantwell, J., Kim, S.H., Schlessinger, J., Zhang, K.Y., West, B.L., Powell, B., Habets, G., Zhang, C., Ibrahim, P.N., Hirth, P., Artis, D.R., Herlyn, M., Bollag, G. Discovery of a selective inhibitor of oncogenic B-Raf kinase with potent antimelanoma activity. Proc. Natl. Acad. Sci. U. S. A. 105, 3041-3046 (2008).

54. Verdonk M.L., Rees D.C., Group efficiency: a guideline for hits-to-leads chemistry. ChemMedChem 2008, 3, 1179-1180.

55. Drysdale, M.J. and Brough, P.A. Medicinal chemistry of Hsp90 inhibitors. Curr. Top. Med. Chem. 8, 859-868 (2008).

56. Murray, C.W., Carr, M.G., Callaghan, O., Chessari, G., Congreve, M., Cowan, S., Coyle, J.E., Downham, R., Figueroa, E., Frederickson, M., Graham, B., McMenamin, R., O'Brien, M.A., Patel, S., Phillips, T.R., Williams, G., Woodhead, A.J., Woolford, A.J. Fragment-based drug discovery applied to Hsp90. Discovery of two lead series with high ligand efficiency. J. Med. Chem. 53, 5942-55 (2010).

57. Woodhead, A.J. Angove, H., Carr, M.G., Chessari, G., Congreve, M., Coyle, J.E., Cosme, J., Graham, B., Day, P.J., Downham, R., Fazal, L., Feltell, R., Figueroa, E., Frederickson, M., Lewis, J., McMenamin, R., Murray, C.W., O'Brien, M.A., Parra, L., Patel, S., Phillips, T., Rees, D.C., Rich, S., Smith, D.M., Trewartha, G., Vinkovic, M., Williams, B., Woolford, A.J. Discovery of (2,4-dihydroxy-5-isopropylphenyl)-[5-(4-methylpiperazin-1ylmethyl)-1,3-dihydrois oindol-2-yl]methanone (AT13387), a novel inhibitor of the molecular chaperone Hsp90 by fragment based drug design. J. Med. Chem. 53, 5956-5969 (2010).

58. Ferenczy,G.G. and Keserü, G.M. How Are Fragments Optimized? A Retrospective Analysis of 145 Fragment Optimizations. J. Med. Chem. 56, 2478-2486 (2013).

59. Jhoti, H., Williams, G., Rees, D.C., Murray, C.W. The 'rule of three' for fragment-based drug discovery: where are we now? Nature Rev. Drug Discov. 12, 644-645 (2013).

60. Vieth, M., Sutherland, J. J. Dependence of Molecular Properties on Proteomic Family for Marketed Oral Drugs. J. Med. Chem. 49, 3451-3453 (2006).

61. Kwong, A. D., Kauffman, R. S., Hurter, P., Mueller, P. Discovery and development of telaprevir: an NS3-4A protease inhibitor for treating genotype 1 chronic hepatitis $C$ virus. Nature Biotechn. 29, 993-1003 (2011).

62. McTigue, M., Murray, B.W., Chen, J.H.; Deng, Y.-L.; Solowiej, J.; Kania, R.S. Molecular conformations, interactions, and properties associated with drug efficiency and clinical performance among VEGFR TK inhibitors. Proc. Natl. Acad. Sci. U. S. A. 109, 18281-18289 (2012).

Lipophilic ligand efficiencies (LLE) of VEGFR tyrosine kinase inhibitors correlate with their clinical efficacy.

63. Davis, M. I., Hunt, J. P., Herrgard, S., Ciceri, P., Wodicka, L. M., Pallares, G., Hocker, M-, Treiber, D. K., Zarrinkar, P. P. Comprehensive analysis of kinase inhibitor selectivity. Nature Biotech. 29, 1046-1051 (2011). 
64. Soth, M., Hermann, J. C., Yee, C., Alam, M., Barnett, J.W., Berry, P., Browner, M.F., Frank, K., Frauchiger, S., Harris, S., et al. 3-Amido Pyrrolopyrazine JAK Kinase Inhibitors: Development of a JAK3 vs JAK1 Selective Inhibitor and Evaluation in Cellular and in Vivo Models. J. Med. Chem. 56, 345-356 (2013).

65. Young, R. J. The successful quest for oral factor Xa inhibitors; learnings for all of medicinal chemistry? Bioorg. Med. Chem. Lett. 21, 6228-35 (2011).

Proposes a general approach to assessing compound quality, exemplified by marketed factor Xa inhibitors, which have lower hydrophobicity and higher ligand efficiencies ( $\left.L E_{A T}\right)$ than other published inhibitors.

66. Lemoine, R.C., Wanner, J. Small Molecule Antagonists of the Chemokine Receptor CCR5. Current Topics in Medicinal Chemistry, 10, 1299-1338 (2010).

67. Cumming, J, G., Tucker, H., Oldfield, J., Fielding, C., Highton, A., Faull, A., Wild, M., Brown, D., Wells, S., Shaw, J. Balancing hERG affinity and absorption in the discovery of AZD5672, an orally active CCR5 antagonist for the treatment of rheumatoid arthritis. Bioorg. Med. Chem. Lett. 22, 1655-1659 (2012).

68. Charles, M. A., Kane, J.P. New molecular insights into CETP structure and function: a review. J. Lipid Res. 53, 1451-1458 (2012).

69. a) Bochem, A. E.; Kuivenhoven, J. A.; Stroes, E. S. G. The promise of cholesteryl ester transfer protein (CETP) inhibition in the treatment of cardiovascular disease. Current Pharmaceutical Design 19, 3143-3149 (2013). b) Mantlo, N.B, Escribano, A. Update on the discovery and development of cholesteryl ester transfer protein inhibitors for reducing residual cardiovascular risk. J. Med. Chem. doi: dx.doi.org/10.1021/jm400574e (2013).

70. Hunt, J.A., Gonzalez, S., Kallashi, F., Hammond, M.L., Pivnichny, J.V., Tong, X., Xu, S.S., Anderson, M.S., Chen, Y, Eveland, S.S., Guo, Q., Hyland, S.A., Milot, D.P., C.P. Sparrow, C.P., Wright, S.D., Sinclair, P.J.. 2Arylbenzoxazoles as CETP inhibitors: Substitution and modification of the $\alpha$-alkoxyamide moiety. Bioorg. Med. Chem. Lett., 20 1019-1022 (2010).

71. Sweis, R. F., Hunt, J. A., Sinclair, P. J., Chen, Y., Eveland, S. S., Guo, Q., Hyland, S. A., Milot, D. P., Cumiskey, A., Latham, M., et al. 2-(4-Carbonylphenyl)benzoxazole inhibitors of CETP: Attenuation of hERG binding and improved HDLc-raising efficacy. Bioorg. Med. Chem. Lett. 21, 2597-2600 (2011).

72. Kallashi, F., Kim, D., Kowalchick, J., Park, Y. J., Hunt, J. A., Ali, A., Smith, C. J., Hammond, M. L., Pivnichny, J. V., Tong, $X$., et al. 2-Arylbenzoxazoles as CETP inhibitors: Raising HDL-C in cynoCETP transgenic mice. Bioorg. Med. Chem. Lett. 21, 558-561 (2011).

73. Harikrishnan, L. S., Kamau, M. G., Herpin, T. F., Morton, G. C., Liu, Y., Cooper, C. B., Salvati, M. E., Qiao, J. X., Wang, T. C., Adam, L. P., et al. 2-Arylbenzoxazoles as novel cholesteryl ester transfer protein inhibitors: Optimization via array synthesis. Bioorg. Med. Chem. Lett. 18, 2640-2644 (2008).

74. Fernanadez , M-C., Escribano, A., Mateo, A. I., Parthasarathy, S., Martin de la Nava, E. M., Wang, X., Cockerham, S. L., Beyer, T.P., Schmidt, R.J., Cao, G., et al. Design, synthesis and structure-activity-relationship of 1,5-tetrahydronaphthyridines as CETP inhibitors. Bioorg. Med. Chem. Lett. 22, 3056-3062 (2012).

75. Harikrishnan,L.S., Finlay, H.J., Qiao, J.X., Kamau, M.G., Jiang, J., Wang, T.C., Li, J., Cooper, C.B., Poss,M.A., Adam, L.P., Taylor,D.S., Chen, A.Y.A., Yin,X., Sleph, P.G., Yang, R.Z., Sitkoff,D. F., Galella, M.A., Nirschl,D.S., Kirk, K.V., Miller,A.V., Huang, C.S., Chang, M., Chen,X-Q., Salvati,M.E., Wexler,R.R., Lawrence, M.R. Diphenylpyridylethanamine (DPPE) Derivatives as Cholesteryl Ester Transfer Protein (CETP) Inhibitors. J. Med. Chem. 55, 6162-6175 (2012).

76. Griffith, D. A., Hadcock, J. R., Black, S. C., Iredale, P. A., Carpino, P. A., DaSilva-Jardine, P., Day, R., DiBrino, J., Dow, R. L., Landis, M. S. Discovery of 1-[9-(4-Chlorophenyl)-8-(2-chlorophenyl)-9H-purin-6-yl]-4 ethylaminopiperidine-4-carboxylic Acid Amide Hydrochloride (CP-945,598), a Novel, Potent, and Selective Cannabinoid Type 1 Receptor Antagonist. J. Med. Chem. 52, 234-237 (2009).

77. Plowright, A. T., Nilsson, K., Antonsson, M., Amin, K., Broddefalk, J., Jensen, J., Lehmann, A., Jin, S., St-Onge, S., Tomaszewski, M.J., et al. Discovery of Agonists of Cannabinoid Receptor 1 with Restricted Central Nervous System Penetration Aimed for Treatment of Gastroesophageal Reflux Disease. J. Med. Chem. 56, 220-240 (2013).

78. Darout,E., Robinson,R.P., McClure,K.F., Corbett,M., Li, B., Shavnya,A., Andrews,M.P., Jones, C.S., Li, Q., Minich,M.L., Mascitti,V., Guimarães, C.R.W., Munchhof, M.J., Bahnck, K.B., Cai,C., Price,D.A., Liras,S., Bonin, P.D., Cornelius,P., Wang,R., Bagdasarian,V., Sobota,C.P., Hornby, S., Masterson,V.M., Joseph,R.M., Kalgutkar, A.S., and Chen, Y.. Design and Synthesis of Diazatricyclodecane Agonists of the G-Protein-Coupled Receptor 119. J. Med. Chem. 56, 301-319 (2013). 
79. Higueruelo, A.P., Schreyer , A., Bickerton, G.R.J., Blundell, T.L., Pitt, W.R. What Can We Learn from the Evolution of Protein-Ligand Interactions to Aid the Design of New Therapeutics? PLOS ONE 7(12), e51742 (doi:10.1371/journal.pone.0051742) (2012).

80. Valko, K., Chiarparin, E., Nunhuck, S., Montanari, D. In vitro measurement of drug efficiency index to aid early lead optimization. J. Pharm. Sci. 101, 4155-4169 (2012).

81. Freeman-Cook, K.D., Hoffman, R.L., Johnson, T.W. Lipophilic efficiency: the most important efficiency metric in medicinal chemistry. Future Med. Chem. 5,113-115 (2013)

82. Abad-Zapatero, C. Ligand efficiency indices for effective drug discovery. Exp. Opin. Drug Disc. 2, 469-488 (2007)

83. Mannhold, R., Poda, G.I., Ostermann, C., Tetko, I.V. Calculation of molecular lipophilicity: state-of-the-art and comparison of logP methods on more than 96,000 compounds. J. Pharm. Sci. 98, 861-893 (2009).

84. Nissink, J. W. M. Simple size-independent measure of ligand efficiency. J. Chem. Inf. Model. 49, 1617-22 (2009).

85. Southan, C., Boppana, K., Jagarlapudi, S.A., Muresan, S. Analysis of in vitro bioactivity data extracted from drug discovery literature and patents: Ranking 1654 human protein targets by assayed compounds and molecular scaffolds. J. Cheminform. 3, 14 (2011).

86. Klibanov, O,M., Williams, S, H., Iler, C. A. Cenicriviroc, an orally active CCR5 antagonist for the potential treatment of HIV infection. Current Opinion in Investigational Drugs 11, 940-950 (2010). 


\section{Box 1 - Ligand efficiency metrics}

Ligand efficiency (LE) was first proposed as a method to compare molecules on the basis of the binding energy per atom (Eq 1a). ${ }^{26,27}$ The concept has since been extended to other properties, including lipophilicity, ${ }^{7}$ molecular weight, polar surface area, ${ }^{82}$ combinations of physical properties, ${ }^{23,48}$ and functional group contributions. ${ }^{54}$ The measures are focused only on in vitro binding affinity and not in vivo properties. Commonly used metrics ( $\mathrm{HA}=$ number of non hydrogen atoms) are shown below (Equations 1a,1c 2-5).

$$
\begin{gathered}
\mathrm{LE}=\Delta \mathrm{G}^{\circ} / \mathrm{HA}=(-2.303 R T / H A) \cdot \log \left(\mathrm{K}_{\mathrm{d}} / \mathrm{C}^{\circ}\right) \\
\text { where } \Delta \mathrm{G}^{\circ}=-2.303 R T \cdot \log \left(\mathrm{K}_{\mathrm{d}} / \mathrm{C}^{\circ}\right)
\end{gathered}
$$

$\Delta H A=(1.37 / L E) p_{d}{ }^{1}-(1.37 / L E) \mathrm{pK}_{d}{ }^{2}$ where $\mathrm{pK}_{\mathrm{d}}{ }^{1}>\mathrm{pK}_{\mathrm{d}}{ }^{2}$

$\mathrm{LE}=(1.37 / \mathrm{HA}) \cdot \mathrm{pIC}_{50}\left(\right.$ or $\left.\mathrm{pK}_{\mathrm{i}}\right)$

$\mathrm{LEI}=\mathrm{pIC}_{50}\left(\mathrm{or} \mathrm{pK} \mathrm{K}_{\mathrm{i}}\right) / \mathrm{HA}$

LLE or LipE $=\mathrm{plC}_{50}\left(\right.$ or $\left.p K_{\mathrm{i}}\right)-\operatorname{cLogP}($ or LogD $)$

$\operatorname{LLE}_{\mathrm{AT}}=0.111+[(1.37 . \mathrm{LLE}) / \mathrm{HA}]$

LELP $=$ cLogP $($ or Log D) / LE

Equation 1a, originally defined by Kuntz et $a{ }^{27}{ }^{27}$ was derived by dividing the Gibbs binding free energy by the number of heavy atom (HA; i.e. non-hydrogen atoms) per molecular to provide LE in units of $\mathrm{kcal} / \mathrm{mole} / \mathrm{heavy}$ atom. The transformation to derive Eq 1a does not violate the logarithm quotient rule. ${ }^{29} \mathrm{R}$ is the ideal gas constant $\left(1.987 \times 10^{-3} \mathrm{kcal} / \mathrm{K} / \mathrm{mol}\right)$ and $\mathrm{T}$ is the temperature in Kelvin $(\mathrm{K}), \mathrm{C}^{\circ}$ is the standard concentration, and $\mathrm{K}_{\mathrm{d}}$ is the binding constant. The assumption is made that the binding free energy is directly proportional to the number of heavy atoms in the ligand. The reference state is commonly assumed to be aqueous solution at $300 \mathrm{~K}, \mathrm{pH}=7$, all other concentrations being $1 \mathrm{M}$. At these standard conditions the term $-2.303 \mathrm{RT}$ is approximately $-1.37 \mathrm{kcal} / \mathrm{mol}$ when the dissociation constant is expressed as the logarithm to base $10\left(\log \mathrm{K}_{\mathrm{d}}\right)$. Changing temperature and standard concentration will change the relative $\Delta \mathrm{G}$. $\mathrm{LE}$ does not state that a change in the heavy atom count of +1 results in a log order change in affinity $\left(\Delta \mathrm{pK}_{d}=1\right) .{ }^{29}$ The linear relation between affinity and number of heavy atoms for a given LE value results from a simple rearrangement of Eq $1 a$ to give the expected HA count increase per log order affinity increase to maintain a constant ligand efficiency at the standard reference state and is shown in Eq $1 \mathrm{~b}$, at the standard reference state, where $\mathrm{pK}_{d}{ }^{1}$ $\mathrm{pK}_{d}^{2}=1$. Although the binding free energy is negative the modulus of LE is commonly presented in the literature. In common practice, the dissociation constant $K_{d}$ is often substituted by $K_{i}$ or IC $C_{50}$ values (Eq 1c). The substitution of $\mathrm{IC}_{50}$ into $\mathrm{Eq} 1 \mathrm{a}$ is technically incorrect as the $\mathrm{IC}_{50}$ value for competitive inhibitors depends 
on the concentration of the competing ligand. Ideally compounds should only be compared when $\mathrm{IC}_{50}$ values are determined under the same assay conditions (in the studies of target ligand efficiencies reported in this paper, checking all assay conditions for $>200,000$ compounds was not practical, but we treat published Ki, $\mathrm{IC}_{50}$ and $\mathrm{EC}_{50}$ values separately). A simple 'ready reckoner' approach has been adopted by some practitioners to define binding efficiency by simply dividing the negative log of the potency measure (divided by the appropriate units of concentration) by size $\left[-\mathrm{plC}_{50} / \mathrm{HA}\right]$ to produce a unitless quantity, the LEI (ligand efficiency index) (Eq 2). Ligand lipophilicity efficiency (LLE, Eq 3) is simply the difference between pActivity and lipophilicity (CLogP or LogD) and is a measure of specificity of binding to the target relative to partitioning into 1-octanol. ${ }^{7}$ LLE is also referred to in the literature as LipE (lipophilic efficiency); ${ }^{43}$ LLE and LipE are defined identically and in this paper we use the term LLE.

Proposed acceptable values of LE and LLE (based on CLogP) for drug candidates are $>\sim 0.3 \mathrm{kcal} / \mathrm{mol} / \mathrm{heavy}$ atom ${ }^{26}$ and $>\sim 5^{7}$ respectively (based on a $<10 \mathrm{nM}$ molecule having HA of $38(\sim 500 \mathrm{Da})^{26}$ and cLogP of $\left.<3^{7}\right)$. Published mean oral drug values provide the benchmark we use for druggability assessment (Figure 4 and Box 4): LE $=0.45$, LLE (based on cLogP ) $=4.43(n=261$, calculated from the supplementary data provided in ref 2). In another compilation ${ }^{44}$ the values were similar: $L E=0.52$, LLE (based on ChemAxon LogP) $=5.02$ $(n=302)$. Supplementary table S1 compiles potencies, LE, LLE and LELP values of exemplar collections of drugs, hits and leads. ${ }^{44}$

Equations (4) and (5) provide efficiency metrics that combine potency, lipophilicity and heavy atom count in different ways, and are useful for fragment optimization. $\operatorname{LLE}_{\mathrm{AT}}(\mathrm{Eq} 4)$ is scaled to be comparable to LE (Eq 1)..$^{48}$ LELP (eq 5) provides a lipophilicity-corrected ligand efficiency metric that indicates the price of ligand efficiency paid in lipophilicity. ${ }^{23}$ Considering the acceptable lower limit of $L E(0.3)$, and the lipophilicity range for lead-like compounds $(-3<\log P<3)$, the optimal LELP value in lead discovery should be in the range $-10<$ LELP $<10{ }^{23}$ It should be noted that LELP values will respond less to size or potency changes as cLogP gets closer to zero. The lipophilicity efficiency metrics LLE, LELP and LLE $E_{A T}$ can be derived using measured 1octanol - water or buffer partition coefficients or with calculated values of LogP or LogD. Because of the risk of variability in calculating lipophilicity, ${ }^{46,83}$ it is recommended that confirmatory experimental data is obtained for exemplar molecules.

Size independent ligand efficiency measures (SILE and FQ) are described in Box 2 and group efficiency (GE) in Box 3. 


\section{Box 2- Size independent measures of ligand efficiency}

Analysis of large numbers of protein-ligand complexes over a wide range of affinities ${ }^{38,39}$ demonstrates that average, or optimal, ligand efficiencies are systematically higher for small ligands than large ligands. In contrast, the relationship between LLE and CLogP is linear, with the slope depending on the extent to which potency is driven by lipophilicity in the ligands used; a slope of -1 indicates no relationship between potency and lipophilicity (supplementary Figure S2 illustrates the non-linear relationship between LE and HA and the linear relationship between LLE and lipophilicity).

LELP and LLE $E_{A T}$ (Box 1) normalise lipophilic efficiency for molecular size. Two size-independent modifications of LE using heavy atom count only have been proposed, namely Fit Quality (FQ) ${ }^{39}$ and Size Independent Ligand Efficiency (SILE). ${ }^{84}$

$\mathrm{FQ}=\left[\mathrm{plC}_{50}\right.$ or $\left.\mathrm{pK}_{\mathrm{i}} \div \mathrm{HA}\right] \div\left[0.0715+(7.5328 \div \mathrm{HA})+\left(25.7079 \div \mathrm{HA}^{2}\right)-\left(361.4722 \div \mathrm{HA}^{3}\right)\right]$

SILE $=\mathrm{pIC}_{50}$ or $\mathrm{pK}_{\mathrm{i}} \div \mathrm{HA}^{0.3}$

FQ normalizes ligand efficiency by binning ligand efficiencies for a large number of disparate complexes and using a scaling factor derived from a spline-fit of the most potent compounds in each bin. SILE does essentially the same with a different fitting function (i.e. $\Delta \mathrm{G} / \mathrm{H} \mathrm{A}^{0.3}$ where $\Delta \mathrm{G}$ stands for the binding free energy). In either case the effect is to transform ligand efficiency into a metric that is more consistent across wide ranges of molecular size. Similarly, this approach has also been applied to derive size independent enthalpy efficiencies, where free energy is replaced by enthalpy. ${ }^{36}$ Of course, this trend begs the question of why the number of heavy atoms isn't a more consistent normalization term for molecular size by itself. At least two factors have been proposed to explain this result. Firstly, the number of non-hydrogen atoms is being used as a surrogate for molecular surface, as the latter would be expected to be more relevant to molecular recognition and binding. Secondly, analysis of computed molecular surface as a function of molecular weight shows that while these two factors are generally related the increase in molecular surface per additional heavy atom declines with increasing size. ${ }^{39}$ This is sensible given that larger molecules by necessity have more buried (i.e. internal) surface relative to small molecules. Thirdly, beyond the breakdown in linearity between number of atoms and surface area, there is also a fundamental problem with satisfying multiple binding sub-pockets simultaneously. Since molecular structures are not infinitely adjustable (i.e. bond distances and angles can only adopt very limited values without introducing strain) structural compromises are increasingly inevitable as ligands become larger. This was demonstrated with simple model systems ${ }^{39}$ and is further supported by subsequent analysis of enthalpy and entropy efficiencies ${ }^{42}$ showing that the size dependency is related to enthalpy (see Supplementary Figure S1). 


\section{Box 3-Group efficiency}

Group Efficiency (GE) ${ }^{54}$ is used to show the contribution that different parts of a lead molecule make to the overall binding affinity. It is analogous to LE but GE refers just to the atoms that have been added onto an existing molecule.

GE gives a guideline for how much gain in affinity should be aimed for given the size of a group that is added to an existing compound. $G E$ is the contribution to the free energy of binding $(\Delta G)$ per heavy atom $(H A$, the number of non hydrogen atoms) of the added group. ${ }^{54}$ So, if a group of atoms is added to compound ' $A$ ' to form compound ' $\mathrm{B}$ ' the GE of the added group is defined by eq 8 :

$\mathrm{GE}=-\Delta \Delta \mathrm{G} / \Delta \mathrm{HA} \quad(8)$

$\Delta \Delta G=\Delta G(B)-\Delta G(A)(9)$

$\Delta H A=H A(B)-H A(A) \quad(10)$

GE is a more sensitive metric than LE when considering the change in affinity as a fragment (or early lead) is grown into a higher affinity and increased molecular weight lead, and it can be used to show 'hot spots' in terms of binding efficiency. Consider, as an example, an early compound, $A$, that has 25 atoms and an affinity of $1 \mu \mathrm{M}$. If a phenyl group is added to compound $A$ and a 10 -fold increase in affinity is measured for the new compound, $B$, the LEs for the two compounds are similar, 0.33 and 0.31 respectively $[A: 6 \times 1.37 / 25$ and $B: 7 \times 1.37 / 31]$. However the GE of the phenyl group is only 0.23 [(7×1.37)- $(6 \times 1.37) / 6]$, indicating that it is a relatively poor addition in terms of binding affinity. As shown in the Table, if a phenyl group $(H A=6)$ is added and if the aim is to achieve GE $=0.39$ (or overall $\mathrm{LE}=0.39$ which is in line with a candidate molecular weight $=400$ and $\left.I C_{50}=10 \mathrm{nM}\right)$, then an affinity increase of 46 -fold is required.

GE is useful in fragment based drug discovery (FBDD) during the optimisation of the binding affinity of a mM or $\mu \mathrm{M}$ fragment into a $\mathrm{nM}$ lead. It provides the chemist with a measure of whether atoms that are added onto the starting fragment justify their existence in terms of binding affinity (see HSP90 example, Figure 3). Some of the limitations of GE are that it can only be determined if binding affinities have been measured for closely related compounds and GE assumes the SAR of the groups being added is additive (ie independent of each other). Furthermore, GE, as with LE and related terms, is primarily intended when focusing on affinity optimisation rather than in vivo properties. 


\begin{tabular}{|c|c|c|c|}
\hline \multirow[b]{2}{*}{$\Delta \mathrm{HA}$} & \multicolumn{3}{|c|}{ Fold-improvement } \\
\hline & $\begin{array}{c}\mathrm{GE}=0.31 \\
(500 \mathrm{MW} \text { lead } \\
\text { with } \mathrm{IC}_{50}= \\
10 \mathrm{nM})\end{array}$ & $\begin{array}{c}\mathrm{GE}=0.39 \\
(400 \mathrm{MW} \text { lead } \\
\left.\text { with } \mathrm{IC}_{50}=10 \mathrm{nM}\right)\end{array}$ & $\begin{array}{c}\mathrm{GE}=0.52 \\
(300 \mathrm{MW} \text { lead } \\
\text { with } \mathrm{IC}_{50}= \\
10 \mathrm{nM})\end{array}$ \\
\hline 1 & 1.7 & 1.9 & 2.3 \\
\hline 2 & 2.8 & 3.6 & 5.5 \\
\hline 3 & 4.6 & 6.8 & 13 \\
\hline 4 & 7.7 & 13 & 30 \\
\hline 5 & 13 & 24 & 71 \\
\hline 6 & 22 & 46 & 170 \\
\hline 7 & 36 & 88 & 390 \\
\hline 8 & 60 & 170 & 920 \\
\hline 9 & 100 & 320 & 2200 \\
\hline 10 & 170 & 600 & 5100 \\
\hline 11 & 280 & 1100 & 12000 \\
\hline 12 & 460 & 2200 & 28000 \\
\hline
\end{tabular}

Box 3 Table. The affinity increase required when adding atoms onto a lead molecule in order to maintain overall Ligand Efficiency. ${ }^{54}$ For example, if a phenyl group, $\mathrm{C}_{6} \mathrm{H}_{5}(\mathrm{HA}=6)$, is added onto a molecule and the resulting affinity increase is 22 -fold this phenyl group has $L E=0.31$. This is referred to as the Group Efficiency (GE). HA = Heavy Atom Count; GE = group efficiency; MW = molecular weight . 


\section{Box 4 - Ligand efficiencies and target druggability}

To examine physical property and ligand efficiency trends at the drug target level, compounds acting at human drug protein targets and their affinities were assembled from the primary medicinal chemical literature using the GVK BIO database.$^{85}$ Important caveats in using the medicinal chemistry literature are that the bulk of the molecules reported are not fully optimised, and in contrast to potency optimisation, physical property-based optimisation is not routine. For targets where mean ligand efficiency values are currently relatively low, better molecules may not yet have been discovered.

The database contained $270,471 \mathrm{~K}_{\mathrm{i}}, \mathrm{IC}_{50}$ and $\mathrm{EC}_{50}$ values from 1,045 targets, predominantly from the Journal of Medicinal Chemistry ( $41 \%$ of values), Bioorganic and Medicinal Chemistry Letters ( $42 \%$ of values) and Bioorganic and Medicinal Chemistry (13\% of values). Where compounds had more than one reported $\mathrm{K}_{\mathrm{i}}, \mathrm{IC}_{50}$ or $\mathrm{EC}_{50}$ value at a specific target, the mean value was determined. The physical properties of all compounds were calculated as described previously, ${ }^{7,17}$ and LE and LLE (based on cLogP) values determined, treating $\mathrm{K}_{\mathrm{i}}$, $\mathrm{IC}_{50}$ and $E \mathrm{C}_{50}$ values separately for each target. Finally weakly active molecules $(>100 \mu \mathrm{M})$ and very large molecules ( $>70$ heavy atoms) were excluded, and the database $(228,265$ compound-assay pairs) was analysed by target-assay type pairs. Of a total of 1690 target-assay pairs only those where there were $\geq 100$ compounds were used, resulting in 480 target-assay pairs covering 329 different targets representing 201,041 molecules ( $88 \%$ of the database; 127 targets had more than one assay type with $\geq 100$ compounds). For the 480 target-assay pairs, median values are: LE 0.32, LLE (based on cLogP) 2.83, HA 29.75, cLogP 3.89.

The median ligand efficiencies of the 480 target-assay pairs span a wide range: $~ 0.2-0.6$ for LE and $-3-9$ for LLE (Figure 4a). This is a consequence of intrinsic target druggability, combined with the properties of the actual molecules synthesised. For the purpose of comparing target class druggabilities only, arbitrarily defined property and efficiency criteria were derived from oral drugs (Figure 4b). For the property criterion, we use the percentage of published molecules at each target that meet both the size and lipophilicity criteria according to the rule of five ( $\mathrm{HA}<38$ (equivalent to molecular weight $<500$ ) and $\mathrm{cLogP}<5$ ). ${ }^{47}$ For the efficiency criterion, we use the percentage of published molecules at each target that have both LE and LLE values greater than oral drug means (LE $>0.45$, LLE $>4.43$ (see Box 1)). Although for the majority of targets, fewer molecules meet the efficiency criteria than the property criteria, both of these measures clearly distinguish relative target class druggabilities. The physical property trends across the target classes in Figure $4 \mathrm{~b}$ are consistent with other studies. ${ }^{17,20,51,60}$ The least druggable target classes, on both property and efficiency measures, are peptidergic and lipidergic G-protein coupled receptors, and nuclear hormone receptors. While the other targets classes have the bulk (>60\%) of their published molecules meeting the rule of five criteria (Figure $4 b$ ), the percentages meeting the drug-like ligand efficiency criteria show greater relative variability, from $2.8 \%$ for kinases to $14.8 \%$ for the most druggable target class, aminergic G-protein coupled receptors. The major challenge in finding active compounds for many targets is shown by the fact 
that 120 of the 480 target assay-pairs have no published compounds with both LE and LLE values exceeding the mean values of oral drugs.

Reducing size and lipophilicity will tend to increase ligand efficiencies, and the individual target data suggest that doing so may not necessarily be detrimental to affinity for many targets. Thus, amongst the 480 targetassay pairs, straight line fits between activity and either heavy atom count or lipophilicity, while mostly statistically significant, frequently have low $r^{2}$ values and the slopes can be negative as well as positive (Figure $4 \mathrm{c}$ and d). In agreement with reported data, ${ }^{2}$ there are broad trends towards increased activity with increasing size and lipophilicity if the full data set of $>200,000$ compounds is analysed without targets being taken into account; the effect of size being greater than lipophilicity. Within individual series of structurally similar compounds typically investigated in lead optimisation, correlations of potency with size and lipophilicity are often found, but when all the published molecules acting at a target are taken into account, increasing these properties does not have a large effect on potency in the majority of cases. The effects of size and lipophilicity are often different on each target as shown by the $r^{2}$ value of 0.14 for the correlation of the slopes of the potency-cLogP versus potency-HA target relationships for the 480 target-assay pairs (see supplementary spreadsheet). A key conclusion follows: for targets that possess small molecule binding sites, there should be no need a priori to seek to increase bulk physical properties in pursuit of increased activity. In practice, optimisation across 59 targets where lipophilic ligand efficiency was used during potency and other optimisations (see Figure 2) resulted in a reduction in mean lipophilicity and an increase in mean size (resulting in an increase in mean LLE, and conservation of mean LE). 


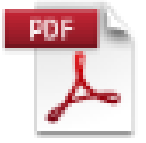

Figures

Figure Captions

Figure 1. Maintaining acceptable ligand efficiencies during optimisation of binding affinity. a) The fold increase in affinity (on the horizontal axis) that is needed to maintain a LE value of 0.3 (Box 1) when adding the indicated groups to a lead molecule. Adding a methyl group $(H A=1)$ requires affinity increase of $\sim 2$-fold whilst adding a benzyl group $(H A=7)$ requires affinity increase of $\sim 30$-fold. b) The fold increase (on the horizontal axis) in binding affinity that is needed to maintain a $L L E_{A T}$ value of 0.3 (Box 1) when adding the indicated groups to an aromatic carbon of a lead molecule. Note the difference in affinity increase required when adding a 6 membered ring according to its cLogP value (piperazine vs morpholine vs pyridine vs phenyl). ${ }^{48}$

Figure 2. Examples from the literature $(n=59)$ where LLE was explicity used in the optimisation process on 47 different targets. Changes in p(Activity) between the starting point and the optimised compound are plotted against the corresponding changes in a) LogP and b) LLE. The upper left quadrant in a) contains examples where activity is increased and cLogP is lowered (34/59 examples). The upper right quadrant in b) contains examples where potency and LLE are increased (48/59 examples). Targets are: 1, 11 beta HSD; 2, ACC1/2; 3, AKT kinase; 4, ASK1; 5, BACE1; 6, CB1; 7, CB2; 8, CCR8; 9, cMet/ALK; 10, DGAT1; 11, Epoxide hydrolase; 12, FFA1/GPR40; 13, FPR1; 14, GP120/CD4; 15, GPR119; 16, GSH-R1a; 17, H4; 18, HIV integrase; 19, HIV RT; 20, HRV VP1; 21, JAK1; 22, LPXC; 23, LRRK2; 24, mGlu1; 25, mGlu2/3; 26, mGluR5; 27, MMP13; 28, MT1/MT2; 29, NMDA GluN2B; 30, N-Myristoyltransferase; 31, NS4B; 32, Ox2; 33, PDE8B; 34, PI3 kinase; 35, PI3K p110b; 36, PIM1; 37, Pin1; 38, PKCO; 39, Progesterone; 40, Tankyrase; 41, TGR5; 42, TYK2; 43, Vasopressin V1a; 44, VEGFR TK; 45, $\beta 1 A R ; 46, \gamma$-secretase; 47, $\sigma 1$. Results from matched pair analysis of starting properties versus optimised properties for the 59 pairs (property, mean difference, $p$ ): Ar ring, +0.14 , 0.172; pActivity, +1.23, <0.0001; cLogP, -0.77, 0.0014; HA, +3.24, <0.0001; Mol Wt, +45.1, <0.0001; TPSA, +19.6, <0.0001; LE, +0.019, 0.077; LLE, +2.00, <0.0001; LELP, -2.45, 0.0018; SILE, +0.50, <0.0001; LLE $_{\mathrm{AT}},+0.77$, $<0.0001$. Full data including literature references are available in the supplementary information spreadsheet.

Figure 3. Example of application of ligand efficiency metrics in fragment-based drug discovery. a) Fragment-to-clinical candidate for HSP90. Some 1600 fragments were screened by NMR LOGSY, and 125 were progressed into X-ray crystallography. ${ }^{56}$ The phenol, 2, has low LE, $0.26 \mathrm{kcal} / \mathrm{mol} /$ heavy atom showing that its binding affinity $(790 \mu \mathrm{M}$ by isothermal titration calorimetry) is suboptimal. However, examination of its binding to HSP9O by X-ray crystallography, together with the binding interactions of previously reported 
chemically related inhibitors (e.g., radicicol), indicated three opportunities for improvement: filling a lipophilic pocket in the region of the methoxy group; incorporating a second hydroxyl group onto the phenol; and growing from the diethylamide into the region occupied by the conformationally flexible Lys 58 side chain. Adding only 6 heavy atoms (non-hydrogen) to phenol 2 led to compound $\mathbf{3}\left(K_{d}=0.54 n M\right)$ whose affinity has increased by $>1,000,000$-fold leading to a corresponding improvement in LE to 0.57 . Optimisation of physicochemical, pharmacokinetic and in vivo properties led to $A T 13387\left(\mathrm{~K}_{d}=0.7 \mathrm{nM}, \mathrm{LE}=\right.$ $0.41)^{57}$, which is currently under evaluation in phase II oncology clinical trials. b) Group Efficiency (GE, Box 3$)^{54}$ for the different parts of the HSP90 inhibitor, AT13387. The GEs are colour coded and illustrate the binding 'hot spots' for this compound. As expected from the fragment-to-lead SAR and the X-ray crystal structure each hydroxyl interacts with the protein via direct and water mediated $\mathrm{H}$-bonds and has very high GE ( $G E=4.65$ and 3.2, corresponding to increase in binding affinity of 2640 fold and 220 fold respectively) while the isopropyl group that fills a lipophilic pocket has high GE (0.79). The piperidine group improves the pharmacokinetic properties without direct binding to HSP90 and hence has a very low GE (-0.04).

Figure 4. Druggability analyses. Data from 480 target-assay pairs with $>100$ compounds covering 329 human drug targets, obtained from the GVK BIO database. ${ }^{85}$ a) Target mean ligand efficiency values are widely distributed. Plot of median target LE versus target LLE (based on CLogP). b) Target class druggability assessment using physical property and ligand efficiency criteria based on marketed drugs. The mean percentage of molecules for each target that meet physical property criteria from the rule of $5^{47}$ is shown, where both cLogP $<5$ and HA $<38$ (HA 38 approximates to molecular weight $500^{26}$ ), versus the mean percentage of molecules for each target that exceed the mean efficiency values of 261 oral drugs (supplementary data of ref 2), where both LLE $\geq 4.43$ and LE $\geq 0.446$. Target classes and numbers of targets in each are shown. Error bars are standard errors of the mean. c), d) Correlations of potency versus size and lipophilicity within targets are often weak. Distribution of the correlation coefficient $\left(r^{2}\right)$ values from straight line fits for the 480 target-assay pairs in a) are shown in c) for pActivity versus heavy atom count and in d) for pActivity versus cLogP . In a) and b), GPCR = G-protein coupled receptor, NHR = nuclear hormone receptor. Data are available in the supplementary information spreadsheet.

Figure 5. Relative ligand efficiencies of $\mathbf{4 6}$ oral drugs acting at $\mathbf{2 5}$ targets. In vitro affinity data for oral drugs ( $\mathrm{IC}_{50}$, Ki or $\mathrm{EC}_{50}$ values) and for other molecules reported in the primary literature to be active at the specific human drug targets were collected from CHEMBL (https://www.ebi.ac.uk/chembl/) in late 2012. Where compounds had $>1$ reported $\mathrm{IC}_{50}$, Ki or $\mathrm{EC}_{50}$ value, the mean was used. Ligand efficiencies and lipophilic ligand efficiencies were calculated (LE and LLE based on CLogP, see Box 1). The $y$-axis shows the \% of compounds reported in the literature acting at the drug target that have both LE and LLE values superior to the oral drug. The targets are arranged on the $x$-axis by target class and show the total numbers of compounds in the analysis. The active form of fingolimod is the SO-phosphate; the parent molecules of the thrombin inhibitor prodrugs melagatran and dabigatran are used. GPCR = G-protein coupled receptor; PDE = phosphodiesterase. Full data are available in the supplementary information spreadsheet. 
Figure 6. Examples of target ligand efficiency analyses. a) LE versus LLE plot for compounds acting at the C$\mathrm{C}$ chemokine receptor type 5 (CCR5, $\mathrm{n}=1513 \mathrm{IC}_{50}$ values). While mean affinity for the target is $<100 \mathrm{nM}$, this comes at some cost in mean physical properties. Mean values of all compounds are: $\mathrm{plC}_{50} 7.57$; HA 38.6; cLogP 4.65; LE 0.27; LLE 2.93. Compounds highlighted are known clinical candidates ${ }^{66,67,86}$ of which only maraviroc (1) has reached the market. AZD $5672^{67}$ was aimed at rheumatoid arthritis, the others at HIV treatment. Cenicriviroc $(6)^{86}$ is a dual CCR5/CCR2 antagonist. The highlighted box shows the compounds that have better combined LE and LLE values than maraviroc ( $1.4 \%$ of the total, the value used for the analysis in Figure 5). AZD5672 (2) is comparable to maraviroc (1) in balancing overall physical properties (especially lipophilicity) and potency. b) Cholesteryl ester transfer protein (CETP, $n=721 I_{50}$ values). The left hand panel, showing the LE versus LLE plot, and the right hand panel, showing the $\mathrm{plC}_{50}$ versus cLogP plot and the LLE=4 boundary, exemplify recommended ligand efficiency data analyses (along with potency versus size) applicable to any target. CETP ${ }^{66,67}$ was chosen because there are several drug candidates in clinical trials and it is one of the most challenging in terms of achieving good physical properties of inhibitors: mean values of all compounds are $\mathrm{pIC}_{50} 6.80$; HA 36.4; CLogP 6.07; LE 0.23; LLE -0.73). The drug candidates have suboptimal LE and especially LLE values. Torcetrapib (7) and dalcetrapib (8; the deacylated active metabolite 9 forms a disulphide bond with Cys-13 on CETP) were discontinued in Phase III because of cardiovascular toxicity and inadequate efficacy respectively. Anacetrapib (10) and evacetrapib (11) are currently in Phase III trials. Even with this poorly druggable target, it is possible to find molecules with better balanced properties, including a class of benzoxazoles (eg 12) ${ }^{70}$ identified from high throughput screening. Notably, compound $13^{74}$ was discovered by lowering the lipophilicity of 7 while retaining comparable affinity, resulting in an increase in LLE of 3.9 units.

$\mathrm{IC}_{50}$ values were taken from CHEMBL (https://www.ebi.ac.uk/chembl/) and LE and LLE (based on cLogP) values were calculated (Box 1 ). The contours represent densities of points.

Figure 7. Explicit use of LLE in optimising compounds acting at the cannabinoid CB1 receptor, where high affinity is often associated with high lipophilicity. In CHEMBL (https://www.ebi.ac.uk/chembl/) there are $3606 \mathrm{pX}_{50}\left(\mathrm{plC}_{50}, \mathrm{pEC}_{50}\right.$ or $\left.\mathrm{pK}_{\mathrm{i}}\right)$ values reported for the $\mathrm{CB1}$ receptor, with the following median values: $\mathrm{pX}_{50}$ 7.1, CLogP 5.8, LLE (based on CLogP) 1.2; only $2.7 \%$ of the compounds have LLE values > 5 . The optimisation examples illustrate that increases of LLE of up to 5 units are possible, even when starting with unpromising leads (LLE <2), without reducing LE. Highlighted areas indicate the design approaches employed, namely conversion of carbon atoms to non-carbon atoms, adding new polar substituents, and removing lipophilic substituents. a) Candidate antagonist 15 from optimisation of lead $14 .^{76}$ b) Agonist 17 with low CNS penetration, derived from screening hit $16 .^{77}$ 


\section{Supplementary Information}

\begin{tabular}{|c|c|c|c|c|c|}
\hline Category $^{44}$ & & pPotency & LE & LLE & LELP \\
\hline \multirow{3}{*}{ Oral Drugs $(n=302)^{a}$} & Mean & 7.76 & 0.52 & 5.02 & 6.38 \\
\hline & Median & 8.09 & 0.47 & 5.02 & 5.74 \\
\hline & SD & 1.63 & 0.21 & 2.12 & 4.90 \\
\hline \multirow{3}{*}{ Phase II $(n=210)^{a}$} & Mean & 8.20 & 0.42 & 5.17 & 8.52 \\
\hline & Median & 8.30 & 0.39 & 5.23 & 7.15 \\
\hline & SD & 1.26 & 0.16 & 2.28 & 7.69 \\
\hline \multirow{3}{*}{$\begin{array}{l}\text { Successful lead } \\
\qquad(n=60)^{b}\end{array}$} & Mean & 6.44 & 0.39 & 3.78 & 8.82 \\
\hline & Median & 6.39 & 0.36 & 3.43 & 8.25 \\
\hline & SD & 1.57 & 0.16 & 2.71 & 10.50 \\
\hline \multirow{3}{*}{ HTS lead $(n=319)^{c}$} & Mean & 7.57 & 0.38 & 3.64 & 11.75 \\
\hline & Median & 7.52 & 0.37 & 3.73 & 10.46 \\
\hline & SD & 0.98 & 0.11 & 1.99 & 7.59 \\
\hline \multirow{3}{*}{ Fragment lead $(n=95)^{d}$} & Mean & 7.09 & 0.37 & 3.91 & 10.24 \\
\hline & Median & 7.22 & 0.34 & 4.24 & 8.21 \\
\hline & SD & 1.38 & 0.12 & 2.53 & 10.03 \\
\hline \multirow{3}{*}{ HTS hit $(n=319)^{c}$} & Mean & 6.18 & 0.35 & 2.51 & 11.98 \\
\hline & Median & 6.14 & 0.34 & 2.42 & 11.13 \\
\hline & SD & 1.02 & 0.12 & 1.83 & 7.51 \\
\hline \multirow{3}{*}{ Fragment hit $(n=100)^{d}$} & Mean & 4.37 & 0.41 & 2.54 & 5.36 \\
\hline & Median & 4.19 & 0.37 & 2.18 & 4.64 \\
\hline & SD & 1.45 & 0.15 & 2.02 & 4.85 \\
\hline
\end{tabular}

Supplementary Table S1. Mean, median and standard deviations (SD) of potencies, LE, LLE and LELP (Box 1) values for collections of oral drugs, Phase II compounds, hits and leads. Compiled in reference 44 (Tarcsay, A., Nyiri, K., Keserü, G. M. Impact of Lipophilic Efficiency on Compound Quality. J. Med. Chem. 55, 1252-1260 (2012)), from cited source data. LLE and LELP are based on logP calculated using ChemAxon, version 5.3.6.. ${ }^{44}$

a. Compiled from https://integrity.thomson-pharma.com/integrity where target affinity was available.

b. Perola, E. An analysis of the binding efficiencies of drugs and their leads in successful drug discovery programs. J. Med. Chem. 53 2986-2997 (2010).

c. Keserü, G. M.; Makara, G. M. The influence of lead discovery strategies on the properties of drug candidates. Nat. Rev. Drug Discovery 8, 203-212 (2009).

d. Alex, A. A.; Flocco, M. M. Fragment-based drug discovery: What has it achieved so far? Curr. Top. Med. Chem. 7, 1544-1567 (2007); Congreve, M.; Chessari, G.; Tisi, D.; Woodhead, A. J. Recent developments in fragment-based drug discovery. J. Med. Chem. 51, 3661-3680 (2008); Schulz, M. N; Hubbard, R. E. Recent progress in fragment-based lead discovery. Curr. Opin. Pharmacol. 9, 1-7. (2009); Murray, C. W.; Rees, D. C. The rise of fragment-based drug discovery. Nat. Chem. 1, 187-19 (2009). 

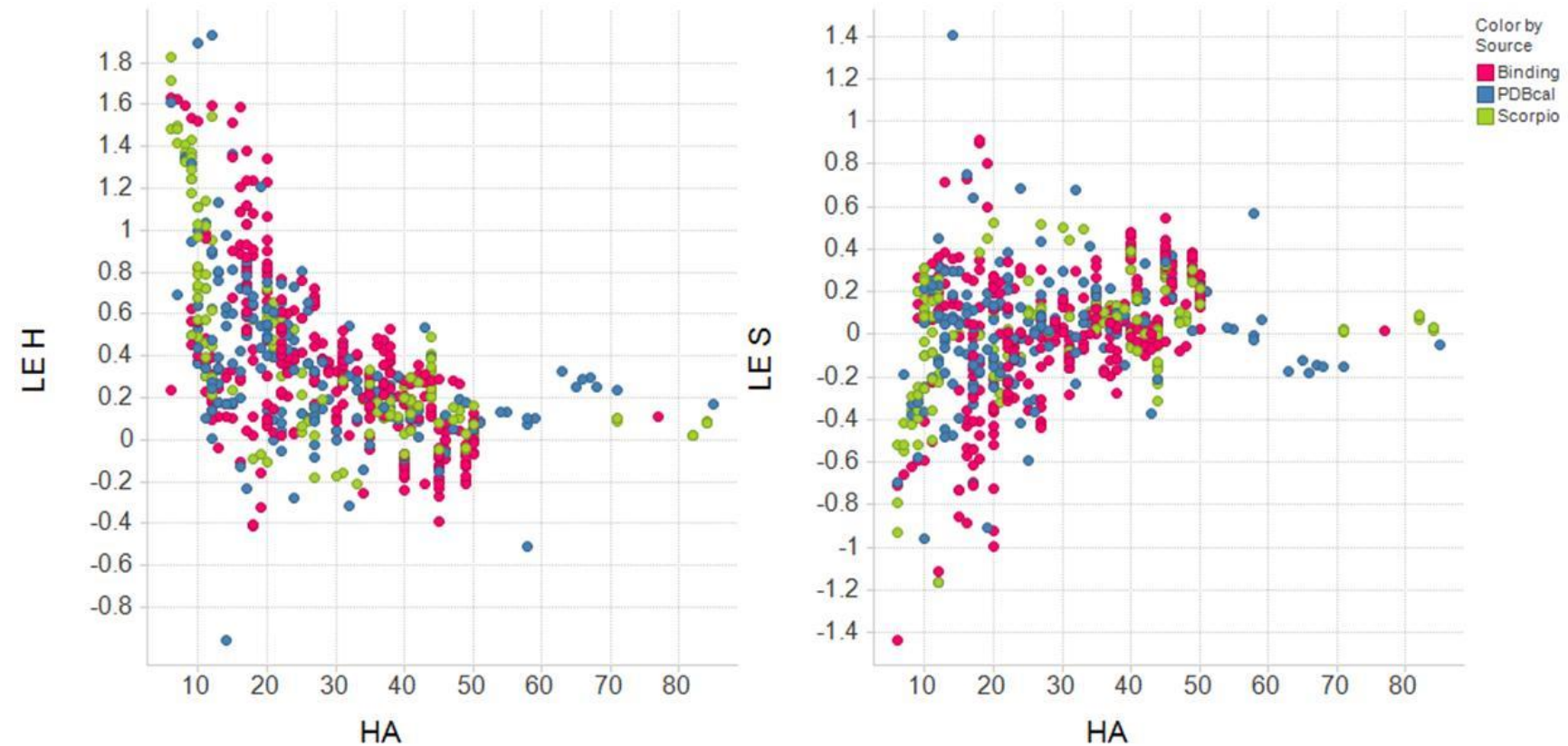

C

d
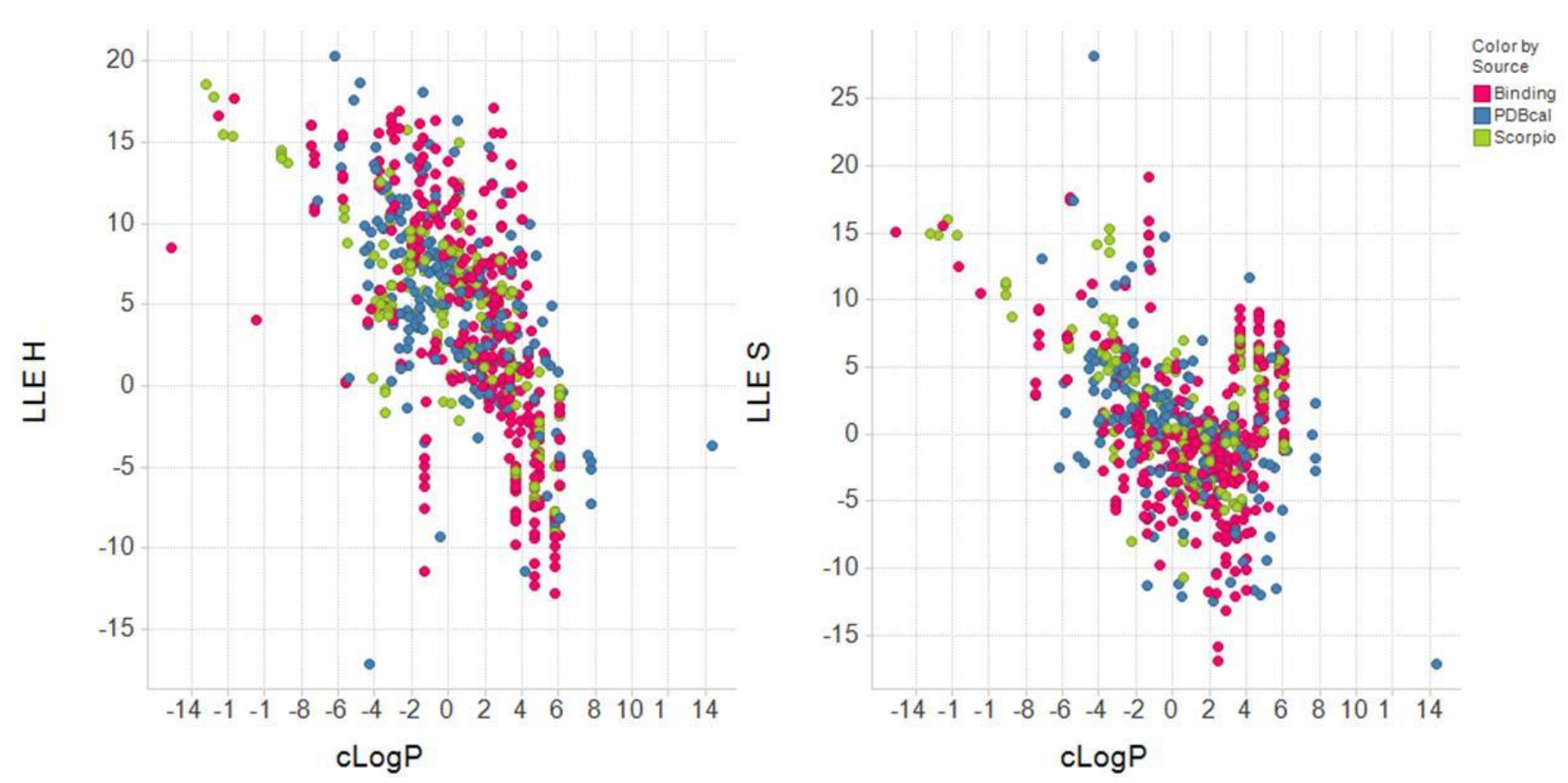

Supplementary Figure S1. Enthalpy and entropy ligand efficiencies versus properties. a) Enthalpy efficiency $\left(L E H=p K_{H}\right.$ $* 1.37 /$ number of heavy atoms), and $b$ ) entropy efficiency (LE $S=\mathrm{pK}_{\mathrm{S}} * 1.37 /$ number of heavy atoms) versus number of heavy atoms. c) Lipophilic enthalpy efficiency (LLE H $=\mathrm{pK}_{\mathrm{H}}$ - cLogP) versus cLogP and d) lipophilic entropy efficiency (LLE $S=p K_{S}$ cLogP) versus cLogP. $\mathrm{pK}_{\mathrm{H}}$ and $\mathrm{pK}_{\mathrm{s}}$ are measures of the enthalpic and entropic components of binding calculated by the formulas of $\frac{-\Delta H}{2.303 R \cdot T}=p K_{d}^{e n t h a l} \stackrel{p y}{=} p K_{H}$ and $\frac{\Delta S}{2.303 R}=p K_{d}^{\text {ent ropy }}=p K_{S}$, respectively. Binding affinity, $\mathrm{pK}_{\mathrm{d}}=\mathrm{pK}_{\mathrm{H}}+\mathrm{pK}_{\mathrm{s}}$. Data ( $n=754)$ are from ref 36 (Ferenczy, G. G., Keserü, G. M. Enthalpic efficiency of ligand binding. J. Chem. Inf. Mod. 50, 1536-1541 (2010)); points are coloured by data sources. 


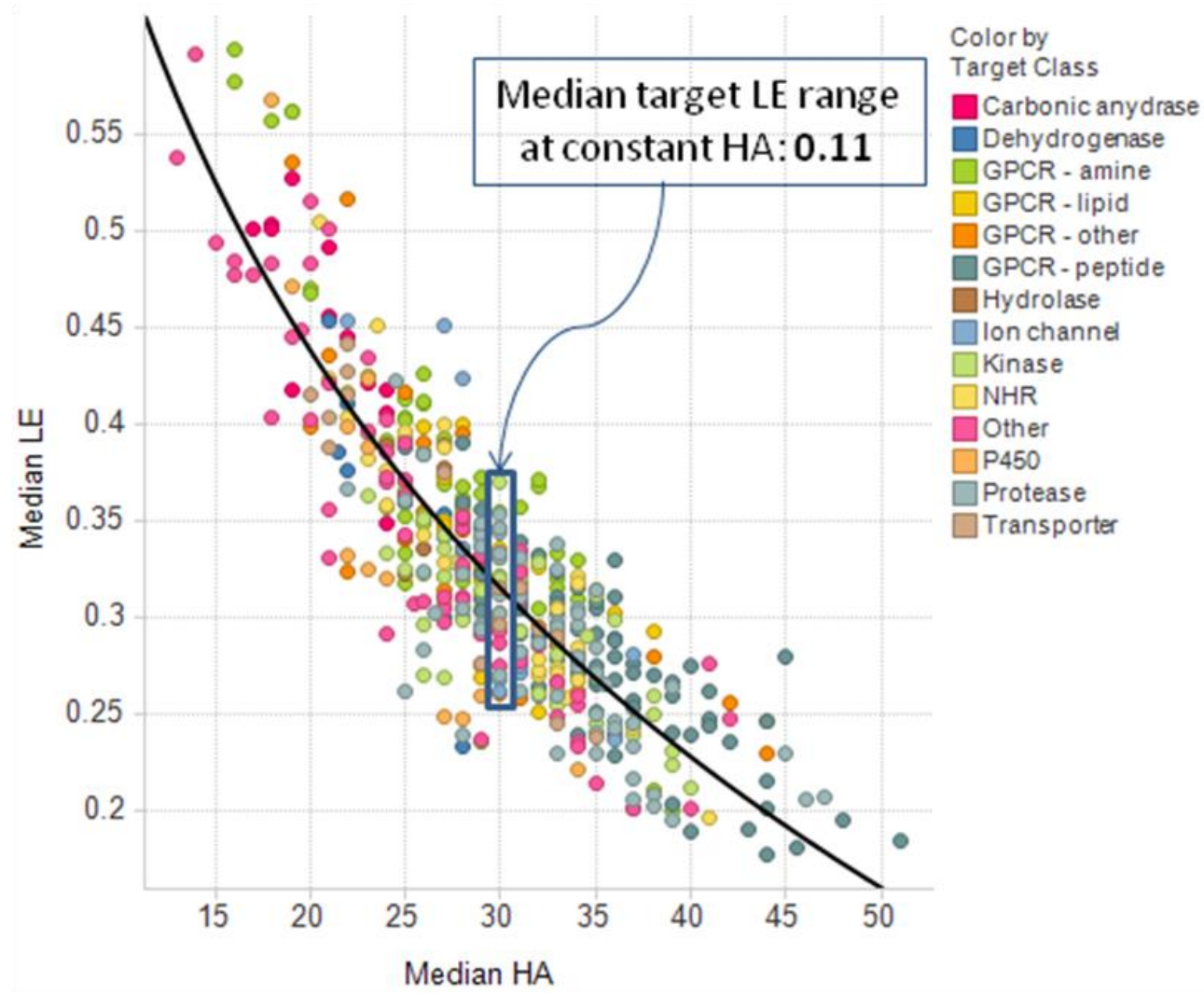

b

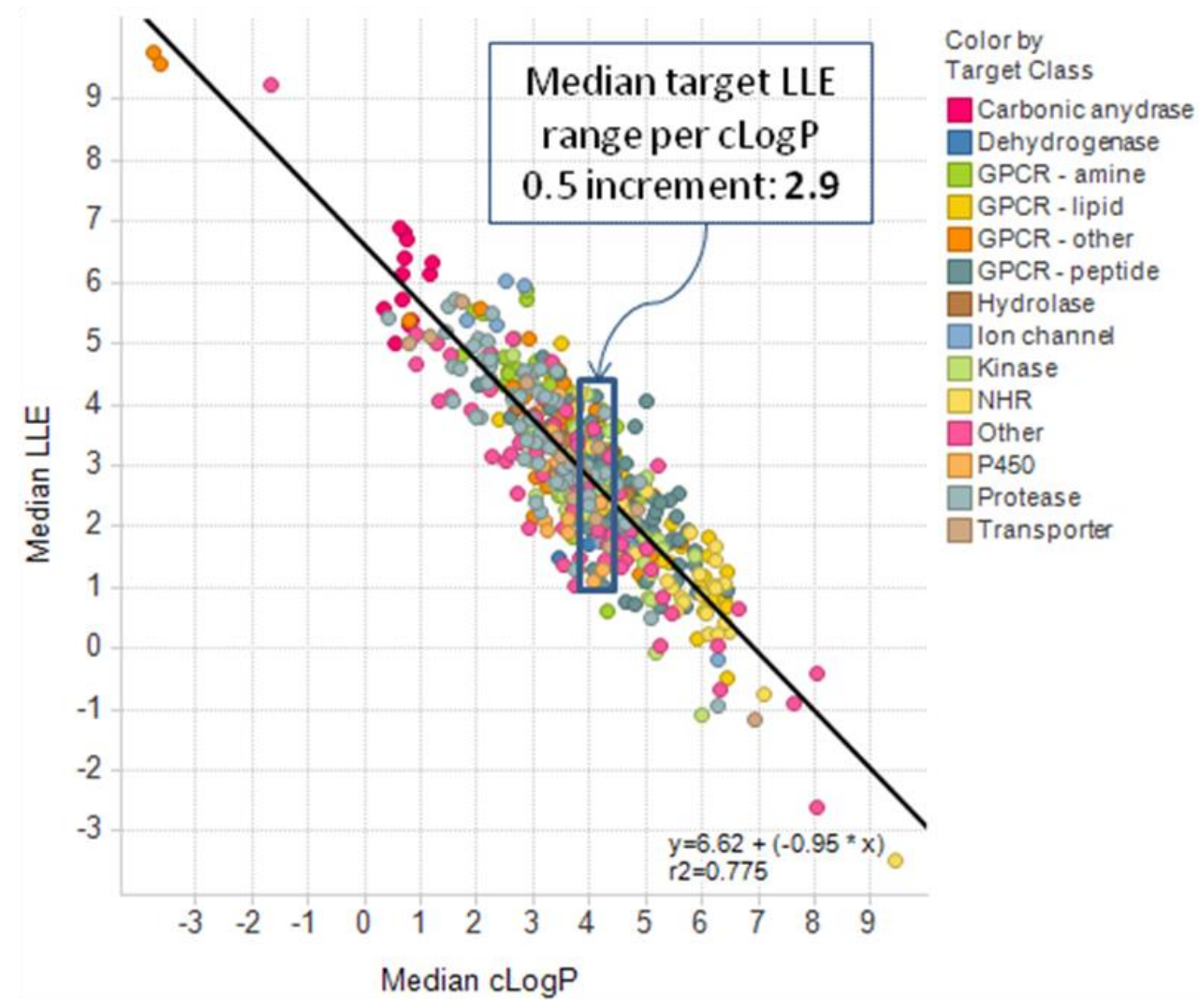

Supplementary Figure S2. Ligand efficiencies versus corresponding physical properties. Relationships between a) LE and HA (non linear, see Box 1) and b) LLE and CLogP, (linear), exemplified by the median values of 480 assay-target pairs (see Figure 4). The approximate ranges of LE and LLE values across targets at fixed values of HA and cLogP is shown. $\mathrm{LE}=$ median target potency $/ \mathrm{HA} \times 1.37$. LLE $=$ median target potency $-\mathrm{cLogP}$. 
Supplementary spreadsheet. Contains: a) Efficiency and property data, and references for the 59 lipophilic optimisations in Figure 2; b) Data for 480 target-assay pairs, including efficiency and property quantiles and statistics of straight line fits of pActivity vs CLogP and HA, used in Figure 4; c) drug efficiencies and property data used to generate Figure 5.

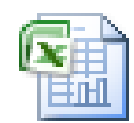

Supplementary

Spreadsheet 